\title{
One-point functions in perturbed boundary conformal field theories
}

\author{
P. Dorey円, M. Pillin民, R. Tateo and G.M.T. Watts \\ ${ }^{1}$ Department of Mathematical Sciences, \\ University of Durham, Durham DH1 3LE, England \\ and \\ SPhT, CEA-Saclay, F-91191 Gif-sur-Yvette Cedex, France \\ ${ }^{2}$ ETAS, PTS-A, Borsigstr. 10, \\ D-70469 Stuttgart, Germany \\ ${ }^{3}$ Universiteit van Amsterdam, Inst. voor Theoretische Fysica \\ 1018 XE Amsterdam, The Netherlands \\ ${ }^{4}$ Mathematics Department, \\ King's College London, Strand, London WC2R 2LS, U.K.
}

\begin{abstract}
We consider the one-point functions of bulk and boundary fields in the scaling Lee-Yang model for various combinations of bulk and boundary perturbations. The one-point functions of the bulk fields are analysed using the truncated conformal space approach and the form-factor expansion. Good agreement is found between the results of the two methods, though we find that the expression for the general boundary state given by Ghoshal and Zamolodchikov has to be corrected slightly. For the boundary fields we use thermodynamic Bethe ansatz equations to find exact expressions for the strip and semi-infinite cylinder geometries. We also find a novel off-critical identity between the cylinder partition functions of models with differing boundary conditions, and use this to investigate the regions of boundary-induced instability exhibited by the model on a finite strip.
\end{abstract}

\footnotetext{
${ }^{1}$ e-mail: P.E.Dorey@durham.ac.uk

${ }^{2}$ e-mail: mathias.pillin@etas.de

${ }^{3}$ e-mail: tateo@wins.uva.nl

${ }^{4}$ e-mail: gmtw@mth.kcl.ac.uk
} 


\section{Introduction}

The purpose of this paper is to make a detailed analysis of the one-point functions in a particular integrable boundary quantum field theory, the boundary scaling Lee-Yang model. Such a study is of intrinsic interest, allowing one to check the consistency of various different approaches to the computation of these quantities. It also serves to illuminate some issues that have arisen in recent investigations of the spectra and boundary entropies ( $g$-functions) of integrable models with boundaries.

After a brief review of the pertinent features of the boundary scaling Lee-Yang model in section 2, sections 3 and 4 of the paper are devoted to the one-point functions of bulk fields in the presence of a boundary. Since these generally have a dependence on the distance from the boundary, their exact determination is a non-trivial problem, of similar difficulty to the calculation of two-point functions in the absence of boundaries. For this reason, apart from some simple cases associated with the Ising model [13], no exact formulae for these boundary one-point functions are known. Instead, one has to resort to approximate methods, two of which we investigate in some detail. The first is the truncated conformal space approach, or TCSA. This has previously been used to study integrable models both without [12, 23] and with [5, 6] boundaries, but this particular application of the technique is new. The second method is based on a form factor (FF) expansion, and makes use of the expansion of the boundary states in the basis of infrared multi-particle states given in [11. Previous work on this topic includes refs. [13, 14, 17, 19]. The main novelties arising in the discussion of the scaling Lee-Yang model presented below are that this model has a non-trivial bulk S-matrix, and that the boundary one-point functions receive contributions from both even- and oddparticle-number components of the boundary state. This allows the relative normalisations of the two sectors to be checked, and we find evidence that the prescription given in [11] is out by a factor of 2. Section 3 introduces and discusses the TCSA and FF methods, culminating in a numerical comparison between the two which, modulo the small correction to the boundary state just mentioned, shows an excellent agreement.

Both the TCSA and the FF methods can be pushed a little further, allowing expectation values in states other than the ground state to be accessed. These generalisations are explained in section 4.

The expectation values of boundary fields are also of interest, and for these there is more hope to find exact results, at least for certain geometries. This is the subject of section 5 . First, in section 5.1, we describe the so-called 'R-channel' approach, which allows us to obtain the expectation values of boundary fields at the edge of a strip of finite width, both in the ground state and, in principle, in any excited state. By relating the expectation values to the derivative of the energy with respect to the boundary field, we find that they can be expressed in terms of the solutions to TBA equations, and compare these results with data from the TCSA (obtained by adapting the method of [12]), for various different combinations of boundary conditions and for varying strip width. Then in section 5.2 we switch to the 'L-channel'. Making use of results from [6], we are able to relate the expectation value of a boundary field placed at one end of a semi-infinite cylinder to the so-called 'Y-function' of the Lee-Yang model. An interesting relationship between the spectra of certain different models placed on the same strip emerges as a by-product of this discussion.

Finally, in section [6, we make use of the results in the preceding sections to examine 
the RG flow from the $\Phi(h)$ boundary to the $\mathbb{1}$ boundary, first discussed in [5], and to treat some previously obscure features of the model on a strip of finite width, with simultaneous perturbations on both boundaries. Section 7 contains our conclusions, and indicates some directions for future work.

As in previous papers [5, 6, 8], we will be using the boundary scaling Lee-Yang model as our example. We have included a brief review of the features of this model in the next section, but the earlier works should be consulted for some more detailed explanations.

\section{The boundary Lee-Yang model}

\subsection{The conformal field theory description of the critical Lee-Yang model}

The Lee-Yang model is the simplest non-unitary conformal field theory, $\mathcal{M}_{2,5}$, and has central charge $-22 / 5$ and effective central charge $2 / 5$. There are only two representations of the Virasoro algebra of interest, of weight 0 and $-1 / 5$, and consequently only two bulk primary fields, the identity $\mathbb{1}$ of weight 0 , and $\varphi$ of weight $x_{\varphi}=-2 / 5$; equally there are only two conformally-invariant boundary conditions, which we denote by $\mathbb{1}$ and $\Phi$.

There are three non-trivial boundary fields $\phi_{-1 / 5}^{(\alpha \beta)}$ interpolating the various boundary conditions $\alpha$ and $\beta$, of weight $h_{\phi}=1 / 5$. Two of these $\left(\psi, \psi^{\dagger}\right)$ interpolate the two different conformal boundary conditions, and one $(\phi)$ lives on the $\Phi$ boundary:

$$
\psi \equiv \phi_{-1 / 5}^{(\mathbb{1} \Phi)}, \quad \psi^{\dagger} \equiv \phi_{-1 / 5}^{(\Phi \mathbb{1})}, \quad \phi \equiv \phi_{-1 / 5}^{(\Phi \Phi)} .
$$

The OPEs of interest are the bulk OPE,

$$
\varphi(z, \bar{z}) \varphi(w, \bar{w})=C_{\varphi \varphi}{ }^{\mathbb{1}}|z-w|^{4 / 5}+C_{\varphi \varphi}^{\varphi}|z-w|^{2 / 5} \varphi(w, \bar{w})+\ldots,
$$

the boundary OPEs,

$$
\begin{aligned}
\phi(z) \phi(w) & =C_{\phi \phi}{ }^{\mathbb{1}}|z-w|^{2 / 5}+C_{\phi \phi}{ }^{\phi}|z-w|^{1 / 5} \phi(w)+\ldots, \\
\psi(z) \phi(w) & =C_{\psi \phi}{ }^{\psi}|z-w|^{1 / 5} \psi(w)+\ldots, \\
\phi(z) \psi^{\dagger}(w) & =C_{\phi \psi^{\dagger}} \psi^{\dagger}|z-w|^{1 / 5} \psi^{\dagger}(w)+\ldots, \\
\psi(z) \psi^{\dagger}(w) & =C_{\psi \psi^{\dagger}} \mathbb{1}|z-w|^{2 / 5}+\ldots, \\
\psi^{\dagger}(z) \psi(w) & =C_{\psi^{\dagger} \psi}{ }^{1}|z-w|^{2 / 5}+C_{\psi^{\dagger} \psi}{ }^{\phi}|z-w|^{1 / 5} \phi(w)+\ldots,
\end{aligned}
$$

and the two bulk-boundary OPEs

$$
\begin{aligned}
& \left.\varphi(z)\right|_{\mathbb{1}}={ }^{(\mathbb{1})} B_{\varphi}^{\mathbb{1}}|2(z-w)|^{2 / 5}+\ldots, \\
& \left.\varphi(z)\right|_{\Phi}={ }^{(\Phi)} B_{\varphi}^{\mathbb{1}}|2(z-w)|^{2 / 5}+{ }^{(\Phi)} B_{\varphi}^{\phi}|2(z-w)|^{1 / 5} \phi(w)+\ldots .
\end{aligned}
$$

\footnotetext{
${ }^{*}$ In [6] we did not distinguish these fields and denoted them both by $\psi$
} 
A suitable choice for the structure constants is

$$
\begin{aligned}
& C_{\varphi \varphi}{ }^{\mathbb{1}}=C_{\phi \phi}{ }^{\mathbb{1}}=-1, \quad C_{\psi \psi^{\dagger}}{ }^{\mathbb{1}}=1, \quad C_{\psi^{\dagger} \psi^{\mathbb{1}}}=-\frac{1+\sqrt{5}}{2}, \\
& C_{\varphi \varphi}{ }^{\varphi}=-\left|\frac{2}{1+\sqrt{5}}\right|^{1 / 2} \cdot \alpha^{2}, \quad{ }^{(\mathbb{1})} B_{\varphi}^{\mathbb{1}}=-\left|\frac{2}{1+\sqrt{5}}\right|^{1 / 2}, \\
& C_{\psi^{\dagger} \psi}{ }^{\phi}=C_{\phi \phi}{ }^{\phi}=-\left|\frac{1+\sqrt{5}}{2}\right|^{1 / 2} \cdot \alpha, \quad{ }^{(\Phi)} B_{\varphi}^{\mathbb{1}}=\left|\frac{1+\sqrt{5}}{2}\right|^{3 / 2}, \\
& C_{\phi \psi \psi^{\dagger}} \psi^{\dagger}=C_{\psi \phi} \psi=-\left|\frac{2}{1+\sqrt{5}}\right|^{1 / 2} \cdot \alpha, \quad{ }^{(\Phi)} B_{\varphi}^{\phi}=\left|\frac{5+\sqrt{5}}{2}\right|^{1 / 2} \cdot \alpha, \\
& \alpha=\left|\frac{\Gamma(1 / 5) \Gamma(6 / 5)}{\Gamma(3 / 5) \Gamma(4 / 5)}\right|^{1 / 2} \text {. }
\end{aligned}
$$

There are three possible choices for pairs of conformal boundary conditions on a strip: $(\mathbb{1}, \mathbb{1})$, $(\Phi, \mathbb{1})$ and $(\Phi, \Phi)$. We shall take the strip to be of width $R$ with coordinates $0 \leq x \leq R$ across the strip and $y$ running along the strip, and normalise all our correlation functions so that the expectation value of the identity operator on a strip is always one.

The strip correlation functions between states $\langle\alpha|$ and $|\beta\rangle$ can be found by mapping the strip to the unit disc and inserting the appropriate fields $\psi_{\alpha}$ and $\psi_{\beta}$. Since the ground state on the strips with boundary conditions $(\mathbb{1}, \mathbb{1}),(\mathbb{1}, \Phi)$ and $(\Phi, \Phi)$ correspond to the fields $\mathbb{1}$, $\psi$ and $\phi$ respectively, one needs to include the appropriate field insertions to find the ground state expectation values on these strips. These insertions lead directly to the particular chiral blocks and structure constants in (2.4) and (2.5).

The one-point functions of the field $\varphi(x)$ on such a strip are best expressed in terms of the four strip chiral block functions $f_{i}(\theta)$,

$$
\begin{aligned}
& f_{1}(\theta)=f_{\mathbb{1}}^{\phi \phi \phi \phi}(\theta)=\left(\frac{2 \sin \theta}{\cos ^{2} \theta}\right)_{2}^{2 / 5} \mathrm{~F}_{1}\left(\frac{4}{10}, \frac{8}{10} ; \frac{11}{10} ;-\tan ^{2} \theta\right) \\
& f_{2}(\theta)=f_{\phi}^{\phi \phi \phi \phi}(\theta)=\left(\frac{2 \sin \theta}{\cos ^{3} \theta}\right)^{1 / 5}{ }_{2} \mathrm{~F}_{1}\left(\frac{3}{10}, \frac{8}{10} ; \frac{9}{10} ;-\tan ^{2} \theta\right), \\
& f_{3}(\theta)=f_{\mathbb{1}}^{\phi \phi \mathbb{1} \mathbb{1}}(\theta)=(2 \sin \theta)^{2 / 5} \\
& f_{4}(\theta)=f_{\phi}^{\phi \phi \phi \mathbb{1}}(\theta)=(2 \sin \theta)^{1 / 5} .
\end{aligned}
$$

In terms of these functions, we have

$$
\begin{aligned}
\langle\varphi(x, y)\rangle_{(\mathbb{1}, \mathbb{1})} & =\left(\frac{R}{\pi}\right)^{2 / 5}{ }^{(\mathbb{1})} B_{\varphi}^{\mathbb{1}} f_{3}\left(\frac{\pi x}{R}\right)={ }^{(\mathbb{1})} B_{\varphi}^{\mathbb{1}}\left(\frac{2 R}{\pi} \sin \frac{\pi x}{R}\right)^{2 / 5}, \\
\langle\varphi(x, y)\rangle_{(\Phi, \mathbb{1})} & =\left(\frac{R}{\pi}\right)^{2 / 5}\left({ }^{(\Phi)} B_{\varphi}^{\mathbb{1}} f_{1}\left(\frac{\pi x}{R}\right)+{ }^{(\Phi)} B_{\varphi}^{\phi} C_{\psi \phi} \psi f_{2}\left(\frac{\pi x}{R}\right)\right) \\
\langle\varphi(x, y)\rangle_{(\Phi, \Phi)} & =\left(\frac{R}{\pi}\right)^{2 / 5}\left({ }^{(\Phi)} B_{\varphi}^{\mathbb{1}} f_{1}\left(\frac{\pi x}{R}\right)+{ }^{(\Phi)} B_{\varphi}^{\phi} C_{\phi \phi} \phi f_{2}\left(\frac{\pi x}{R}\right)\right) .
\end{aligned}
$$

It is only for the latter two pairs of boundary conditions that the boundary field $\phi(y)$ exists on the boundary $x=0$. For these cases the one-point functions are simply

$$
\langle\phi(y)\rangle_{(\Phi, \mathbb{1})}=\left(\frac{R}{\pi}\right)^{1 / 5} C_{\psi \phi} \psi, \quad\langle\phi(y)\rangle_{(\Phi, \Phi)}=\left(\frac{R}{\pi}\right)^{1 / 5} C_{\phi \phi} \phi .
$$


Of the five expectation values (2.4), 2.5), only the first has a finite limit as the strip width tends to $\infty$, the others all diverging. None of them depend on $y$, and so when no confusion can arise this variable will often be omitted, even inside the vacuum expectation values.

\subsection{The scaling Lee-Yang model}

The scaling Lee-Yang (SLY) model can be described as a perturbation of the critical Lee-Yang model by the term

$$
\lambda \int \varphi(w, \bar{w}) \mathrm{d}^{2} w .
$$

This leads to a massive scattering theory, comprising a single particle with two-particle $\mathrm{S}_{-}$ matrix [ []

$$
S(\theta)=-(1)(2), \quad(x)=\frac{\sinh \left(\frac{\theta}{2}+\frac{i \pi x}{6}\right)}{\sinh \left(\frac{\theta}{2}-\frac{i \pi x}{6}\right)} .
$$

The mass $M$ of the particle is related to the bulk perturbation parameter $\lambda$ by [24, 26]

$$
M=\kappa \lambda^{5 / 12}, \quad \kappa=2^{19 / 12} \sqrt{\pi} \frac{(\Gamma(3 / 5) \Gamma(4 / 5))^{5 / 12}}{5^{5 / 16} \Gamma(2 / 3) \Gamma(5 / 6)}=2.642944 \ldots .
$$

We will also need the form factors of the bulk model. These are the matrix elements of the elementary field $\varphi(x)$ in the asymptotic $n$-particle states which can be formally written in terms of the ZF operators $A(\theta)$ as $\left|\theta_{1}, \ldots, \theta_{n}\right\rangle=A\left(\theta_{1}\right) \ldots A\left(\theta_{n}\right)|0\rangle$. The form-factor $F_{n}\left(\theta_{i} \ldots \theta_{n}\right)$ is then given by

$$
\left\langle 0|\varphi(x, 0)| \theta_{1}, \ldots, \theta_{n}\right\rangle=\exp \left(-M x \sum_{i} \cosh \theta_{i}\right) F_{n}\left(\theta_{1}, \ldots, \theta_{n}\right) .
$$

The form factors of the SLY model were first computed in [21]; we, however, adopt the conventions of [25], modulo the fact that for us $\varphi(x)$ is a real field. The function $F_{n}$ can be parametrised as [25]:

$$
F_{n}\left(\theta_{1}, \ldots, \theta_{n}\right)=H_{n} Q_{n}\left(x_{1}, \ldots, x_{n}\right) \prod_{i<j}^{n} \frac{f\left(\theta_{i}-\theta_{j}\right)}{x_{i}+x_{j}},
$$

where $x_{i}=\exp \left(\theta_{i}\right), i=1, \ldots, n$. The terms in (2.10) can be determined through the form factor bootstrap [22], with the result

$$
f(\theta)=\frac{\cosh \theta-1}{\cosh \theta+1 / 2} v(i \pi-\theta) v(-i \pi+\theta),
$$

where we take the function $v$ in a form suitable for numerical evaluation 25

$$
\begin{aligned}
v(\theta) & =\prod_{n=1}^{N}\left[\frac{\left(\frac{\theta}{2 \pi i}+n+1 / 2\right)\left(\frac{\theta}{2 \pi i}+n-1 / 6\right)\left(\frac{\theta}{2 \pi i}+n-1 / 3\right)}{\left(\frac{\theta}{2 \pi i}+n-1 / 2\right)\left(\frac{\theta}{2 \pi i}+n+1 / 6\right)\left(\frac{\theta}{2 \pi i}+n+1 / 3\right)}\right]^{n} \\
& \times \exp \left(2 \int_{0}^{\infty} \mathrm{d} t \frac{\sinh (t / 2) \sinh (t / 3) \sinh (t / 6)}{t \sinh ^{2}(t)}\left(N+1-N \mathrm{e}^{-2 t}\right) \mathrm{e}^{-2 N t+i \theta t / \pi}\right), \\
v(0) & =1.111544045 \ldots
\end{aligned}
$$


(with $N$ arbitrary) and

$$
H_{n}=\psi\left(-\frac{i 3^{1 / 4}}{\sqrt{2} v(0)}\right)^{n}
$$

This overall normalisation of the form factors is taken from the results of [10], where (in our conventions) the expectation value $\langle\varphi\rangle$ in the bulk is

$$
\begin{aligned}
\psi=\langle\varphi\rangle & =-|\lambda|^{-1 / 6} \frac{5^{1 / 6} \Gamma(1 / 3)^{2}}{2^{7 / 4} \cdot 3^{1 / 2} \cdot \pi^{7 / 6}} \frac{\Gamma(1 / 6)}{\Gamma(5 / 6)}\left[\frac{\Gamma(9 / 10)}{\Gamma(1 / 10)}\right]^{5 / 12} \\
& =(-0.840184 \ldots)|\lambda|^{-1 / 6}
\end{aligned}
$$

Using the relation between $M$ and $\lambda$ in (2.8), this boils down to

$$
\psi=\frac{-3^{\frac{9}{10}} \Gamma\left(\frac{1}{3}\right)^{\frac{36}{5}} M^{-\frac{2}{5}}}{(2 \pi)^{\frac{14}{5}} 5^{\frac{1}{4}} \Gamma\left(\frac{1}{5}\right) \Gamma\left(\frac{2}{5}\right)}=(-1.239394325 \ldots) M^{-2 / 5} .
$$

The functions $Q_{n}\left(x_{1}, \ldots, x_{n}\right)$ in (2.10) are symmetric polynomials of degree $n(n-1) / 2$ and partial degree $n-1$. These polynomials have been determined via the form factor bootstrap approach for arbitrary particle numbers $n$. They can be nicely written in the form of a determinant of a matrix in symmetric polynomials [21,25], for a related formulation see [20]. For our purposes it will be sufficient to list the first few:

$$
Q_{0}=1, Q_{1}=1, Q_{2}=e_{1}^{(2)}, Q_{3}=e_{2}^{(3)} e_{1}^{(3)}, Q_{4}=e_{3}^{(4)} e_{2}^{(4)} e_{1}^{(4)},
$$

where the elementary symmetric polynomials in $n$ variables $e_{r}^{(n)}$ are defined by

$$
\prod_{i=1}^{n}\left(1+t x_{i}\right)=\sum_{r=0}^{n} t^{r} e_{r}^{(n)}
$$

The integrable boundary conditions for the model were discussed in detail in [5]. The allowed boundary conditions are the $\mathbb{1}$ conformal b.c., and the perturbation $\Phi(h)$ of the conformal $\Phi$ boundary by the integral along the boundary

$$
h \int \phi(x) \mathrm{d} x .
$$

The boundary reflection factors corresponding to these two boundary conditions are

$$
R_{\Phi(h)}(\theta)=R_{b}(\theta), \quad R_{\mathbb{1}}(\theta)=R_{0}(\theta),
$$

where

$$
R_{b}(\theta)=\left(\frac{1}{2}\right)\left(\frac{3}{2}\right)\left(\frac{4}{2}\right)^{-1}\left(S\left(\theta+i \pi \frac{b+3}{6}\right) S\left(\theta-i \pi \frac{b+3}{6}\right)\right)^{-1} .
$$

The relation between $b$ and $h$ was conjectured in [5] to be

$$
h(b)=-\left|h_{\mathrm{crit}}\right| \sin (\pi(b+1 / 2) / 5) .
$$


Sometimes we shall find it useful to consider instead the dimensionless quantity

$$
\widehat{h}_{\text {crit }}=h_{\text {crit }} M^{-6 / 5}
$$

a constant which was found in [6]:

$$
\widehat{h}_{\text {crit }}=-\pi^{3 / 5} 2^{4 / 5} 5^{1 / 4} \frac{\sin \frac{2 \pi}{5}}{\left(\Gamma\left(\frac{3}{5}\right) \Gamma\left(\frac{4}{5}\right)\right)^{1 / 2}}\left(\frac{\Gamma\left(\frac{2}{3}\right)}{\Gamma\left(\frac{1}{6}\right)}\right)^{6 / 5}=-0.68528998399118 \ldots .
$$

Combining (2.8) and (2.21), we obtain the more convenient formula

$$
h=-\frac{5^{3 / 8}}{2^{1 / 2}} \frac{\sin (\pi(b+1 / 2) / 5)}{\sin (\pi / 5)} \lambda^{1 / 2} .
$$

Finally, we will need the boundary-particle couplings $g_{\alpha}^{a}$ for the various boundary conditions. In [11] these were defined in two different ways, either via the residue at $\theta=i \pi / 2$ of the reflection factor $R_{\alpha}^{a}(\theta)$ for a particle of type $a$ on the boundary $\alpha$ :

$$
R_{\alpha}^{a}(\theta) \sim \frac{i}{2} \frac{\left(g_{\alpha}^{a}\right)^{2}}{\theta-i \pi / 2}
$$

or, in models where bulk fusings occur, via the residues of certain other poles, divided by the corresponding bulk couplings. (If the boundary scattering is non-diagonal, the formulae become a little more complicated - see [11].) For the Lee-Yang model, the consistency of the two definitions was shown to follow from the bootstrap equations and crossing in [8]. Since for this case there is only one particle type, the particle index $a$ can be dropped, and we have

- For the $\mathbb{1 1}$ boundary,

$$
g_{\mathbb{1}}=-i 2 \sqrt{2 \sqrt{3}-3} \text {. }
$$

- For the $\Phi(h(b))$ boundary,

$$
g_{\Phi}(b)=\frac{\tan ((b+2) \pi / 12)}{\tan ((b-2) \pi / 12)} g_{\mathbb{1}} .
$$

Notice that while the reflection factors for the $\mathbb{1}$ and $\Phi(h(0))$ are identical, the corresponding boundary-particle couplings differ by a sign. This can be traced to the fact that when $b=0$, the residue of $R_{b}$ at $i \pi / 6$ for the $\Phi(h(0))$ boundary receives additional contributions from intermediate states containing boundary bound states. The net effect is to negate the coupling; indeed, this is the only option given that the residue at $i \pi / 2$ must remain unchanged. In terms of the boundary states to be discussed in the next section, this means that the only difference between the (infinite-volume) boundary states for these two boundary conditions is in the sign of the contributions from states of odd particle number. 


\section{The one-point functions of the bulk field}

\section{$3.1\langle\varphi(x)\rangle$ from form factors}

In this section we consider the one-point function $\langle\varphi(x)\rangle$ in the presence of a boundary using the form factor approach. We will restrict consideration to a theory with a single scalar particle, as in the Lee-Yang model. Schematically, the idea of the form-factor approach is to evaluate the one-point function on the upper half plane as

$$
\langle\varphi(x)\rangle=\left\langle 0|\varphi(x)| B_{\alpha}\right\rangle=\sum_{n=0}^{\infty}\langle 0|\varphi(x)| n\rangle\left\langle n \mid B_{\alpha}\right\rangle,
$$

where $\left|B_{\alpha}\right\rangle$ is a boundary state corresponding to the boundary condition $\alpha$ and the sum over asymptotic states has been split into the contributions from $n=0,1,2, \ldots$ particles (here $|n\rangle\langle n|$ represents the projection onto asymptotic states with $n$ particles). We have also taken the boundary state to be normalised such that $\left\langle 0 \mid B_{\alpha}\right\rangle=1$.

This boundary state can be expanded in terms of multi-particle states on the infinite line, using the Zamolodchikov-Faddeev (ZF) operators $A(\theta)$ which create single particles of rapidity $\theta$. When the state contains no zero-rapidity particles, it can be written as [1]

$$
\left|B_{\alpha}\right\rangle=\exp \left[\int_{-\infty}^{\infty} \frac{\mathrm{d} \theta}{4 \pi} K_{\alpha}(\theta) A(-\theta) A(\theta)\right]|0\rangle
$$

where $K_{\alpha}(\theta)$ is related to the reflection amplitude $R_{\alpha}(\theta)$ for the $\alpha$ boundary condition by

$$
K_{\alpha}(\theta)=R_{\alpha}\left(\frac{i \pi}{2}-\theta\right)
$$

In general there may also be contributions to the boundary state involving zero-momentum particles, which can be associated with couplings of single bulk particles to the boundary. Up to the three-particle contribution, the appropriate boundary state was given in [1] as

$$
\begin{aligned}
\left|B_{\alpha}\right\rangle & =\left[1+\widetilde{g}_{\alpha} A(0)+\int_{-\infty}^{\infty} \frac{\mathrm{d} \theta}{4 \pi} K_{\alpha}(\theta) A(-\theta) A(\theta)\right. \\
& \left.+\tilde{g}_{\alpha} A(0) \int_{-\infty}^{\infty} \frac{\mathrm{d} \theta}{4 \pi} K_{\alpha}(\theta) A(-\theta) A(\theta)+\ldots\right]|0\rangle,
\end{aligned}
$$

and it is natural to suppose that the full expression is

$$
\left|B_{\alpha}\right\rangle=\exp \left[\tilde{g}_{\alpha} A(0)+\int_{-\infty}^{\infty} \frac{\mathrm{d} \theta}{4 \pi} K_{\alpha}(\theta) A(-\theta) A(\theta)\right]|0\rangle .
$$

In [11], the factor $\widetilde{g}_{\alpha}$ was identified with the boundary-particle coupling $g_{\alpha}$, as defined at the end of the last section. However, our numerical results (and also an examination of the reflection factor and boundary state for the Ising model with free boundary conditions given in [11]) cast doubt on this suggestion. As will be explained in section 3.3 below, we found that we had rather to set

$$
\widetilde{g}_{\alpha}=g_{\alpha} / 2
$$


in order to obtain a successful match with TCSA data.

The form factors for the Lee-Yang model were given in the previous section, and substituting these into (3.3), we find the leading large- $x$ behaviour of $\langle\varphi(x)\rangle$ is

$$
\begin{aligned}
\langle\varphi(x)\rangle & =\left\langle 0|\varphi(x)| B_{\alpha}\right\rangle \\
& =\psi\left(1-i \widetilde{g}_{\alpha} \frac{3^{1 / 4}}{\sqrt{2} v(0)} \mathrm{e}^{-M x}-\frac{3^{1 / 2}}{2 v(0)^{2}} \int_{-\infty}^{\infty} \frac{\mathrm{d} \theta}{4 \pi} K_{\alpha}(\theta) f(-2 \theta) \mathrm{e}^{-2 M x \cosh (\theta)}\right. \\
+i \widetilde{g}_{\alpha} & \left.\frac{3^{3 / 4}}{2 \sqrt{2} v(0)^{3}} \int_{-\infty}^{\infty} \frac{\mathrm{d} \theta}{4 \pi} K_{\alpha}(\theta) \frac{f(\theta) f(-\theta) f(-2 \theta)(1+2 \cosh \theta)^{2}}{8 \cosh \theta \cosh (\theta / 2)^{2}} \mathrm{e}^{-M x(1+2 \cosh (\theta))}+\ldots\right)
\end{aligned}
$$

This will be compared with TCSA data in section 3.3 below.

\subsection{Estimating $\langle\varphi(x)\rangle$ using the TCSA}

The first step in the calculation of $\langle\varphi(x)\rangle$ using the TCSA is the numerical evaluation of the ground state $|\widehat{0}\rangle$ of the perturbed Hamiltonian with dimensionless strip width $r \equiv M R$.

$$
\begin{aligned}
& \widehat{H}\left(R, \lambda, h_{l}, h_{r}\right) \\
= & \frac{\pi}{R}\left(L_{0}-\frac{c}{24}+\lambda\left|\frac{R}{\pi}\right|^{12 / 5} \int_{\theta=0}^{\pi} \widehat{\varphi}(\exp (i \theta)) \mathrm{d} \theta+h_{l}\left|\frac{R}{\pi}\right|^{6 / 5} \widehat{\phi}_{l}(-1)+h_{r}\left|\frac{R}{\pi}\right|^{6 / 5} \widehat{\phi}_{r}(1)\right),
\end{aligned}
$$

where $\widehat{\mathcal{O}}$ represents the operator $\mathcal{O}$ on the upper half plane restricted to the conformal space truncated to level $N$. The parameter $\lambda$ determines the bulk mass and we have allowed the possibility of boundary fields on the left and right edges of the strip, with strengths $h_{l}$ and $h_{r}$. The second step is then to estimate the expectation value of the (dimensionless) operator $M^{2 / 5} \varphi(x)$ in terms of the matrix elements of the operator $\widehat{\varphi}$ as

$$
\left\langle M^{2 / 5} \varphi(x)\right\rangle \sim\left(\frac{r}{\pi}\right)^{2 / 5} \frac{\langle\widehat{0}|\widehat{\varphi}(\exp (i \pi x / R))| \widehat{0}\rangle}{\langle\widehat{0} \mid \widehat{0}\rangle} .
$$

The state $|\widehat{0}\rangle$ can be expanded in Virasoro primary and descendent states; by repeatedly commuting Virasoro algebra elements through $\widehat{\varphi}$, the general matrix elements of $\widehat{\varphi}$ can be expressed in terms of the matrix elements between Virasoro primary states, and their derivatives. In the rest of this section we discuss the TCSA method in more detail; a comparison of the TCSA and FF results is given in section 3.3. All the TCSA results in this paper were calculated on a workstation in Mathematica with truncation levels up to 18 and on spaces with up to 161 states. 


\subsubsection{The strip with $(\mathbb{1}, \mathbb{1})$ boundary conditions}

The simplest case is the strip with boundary conditions $\mathbb{1}$ on both sides. The unperturbed conformal field theory expectation value is given in (2.4). If we denote the scaled position of the field by $\xi$ and the normalised strip width by $r$ where

$$
\xi=x M, \quad r=M R, \quad \text { so that } 0 \leq \xi \leq r,
$$

then the TCSA estimate of the expectation value in the bulk perturbed model truncated to level $N$ takes the form

$$
\left.\mathcal{G}^{(N, r)}(\xi) \equiv M^{2 / 5}\langle\varphi(x)\rangle_{(\mathbb{1}, \mathbb{1})}\right|_{N, r}=\left(\frac{2 r}{\pi} \sin \frac{\pi \xi}{r}\right)^{2 / 5} \sum_{n=0}^{N} f_{n}^{(N, r)} \cos \left(\frac{2 n \pi \xi}{r}\right) .
$$

The coefficients $f_{n}^{(N, r)}$ are determined by the expansion of the ground state $|\widehat{0}\rangle$ and have to be calculated numerically. Since the state $|\widehat{0}\rangle$ lies in the $h=0$ representation, the matrix elements of $\widehat{\varphi}$ are given in terms of the chiral block $f_{3}$; furthermore, since this representation has a null state at level 1 , one can eliminate all states containing $L_{-1}$, and so one does not need any terms with derivatives of this chiral block in (3.8). To compare with the form-factor calculation we will need to take the simultaneous limits $N \rightarrow \infty, r \rightarrow \infty$ while keeping $\xi$ fixed. We shall often drop the labels $(N, r)$ and write simply $\mathcal{G}(\xi)$ for the TCSA estimates.

To show the typical behaviour of these quantities, we give the values of $f_{n}^{(N, r)}$ for $r=8$ and $4 \leq N \leq 12$ in table 1, and for $N=12$ and $2 \leq r \leq 12$ in table 2. In figure 1 we plot $\mathcal{G}^{(12, r)}(\xi)$ for fixed truncation level $N=12$ and for varying values of $r$. We see that on increasing $r, \mathcal{G}^{(12, r)}(\xi)$ approaches a universal form until truncation effects take over and the TCSA approximation breaks down.

There are two sources of error in the TCSA estimate $\mathcal{G}^{(N, r)}(\xi)$. Firstly, TCSA gives the function $\mathcal{G}^{(N, r)}(\xi) \times((2 l / \pi) \sin (\pi \xi / l))^{-2 / 5}$ in equation (3.8) as a Fourier series truncated at the $2 N^{\text {th }}$ term, which leads to the usual errors associated with truncation of Fourier series. Secondly, the coefficients $f_{n}^{(N, r)}$ appearing in the truncated Fourier series are only

\begin{tabular}{|c|c|c|c|c|c|}
\hline$n$ & $\begin{array}{c}N \\
4\end{array}$ & 6 & 8 & 10 & 12 \\
\hline 0 & -0.695639 & -0.695205 & -0.695043 & -0.694975 & -0.694979 \\
\hline 1 & -0.074584 & -0.072820 & -0.072397 & -0.072162 & -0.072053 \\
\hline 2 & -0.015439 & -0.013388 & -0.003932 & -0.012698 & -0.012618 \\
\hline 3 & -0.0004456 & -0.004562 & -0.009036 & -0.003828 & -0.003715 \\
\hline 4 & $-4.406 \cdot 10^{-5}$ & -0.0001517 & -0.001821 & -0.001572 & -0.001524 \\
\hline 5 & & $-2.009 \cdot 10^{-5}$ & $-6.377 \cdot 10^{-5}$ & -0.002017 & -0.0007604 \\
\hline 6 & & $-3.59 \cdot 10^{-6}$ & $-9.89 \cdot 10^{-6}$ & $-3.15 \cdot 10^{-5}$ & -0.000480 \\
\hline 7 & & & $-2.30 \cdot 10^{-6}$ & $-5.29 \cdot 10^{-6}$ & $-1.74 \cdot 10^{-5}$ \\
\hline 8 & & & $-5.60 \cdot 10^{-7}$ & $-1.43 \cdot 10^{-6}$ & $-3.06 \cdot 10^{-6}$ \\
\hline 9 & & & & $-4.42 \cdot 10^{-7}$ & $-8.95 \cdot 10^{-7}$ \\
\hline 10 & & & & $-1.29 \cdot 10^{-7}$ & $-3.20 \cdot 10^{-7}$ \\
\hline 11 & & & & & $-1.17 \cdot 10^{-7}$ \\
\hline 12 & & & & & $-3.84 \cdot 10^{-8}$ \\
\hline
\end{tabular}
approximately calculated.

Table if:

The TCSA coefficients $f_{n}^{(N, r)}$ for $r=8$ and $4 \leq N \leq 12$. 


\begin{tabular}{|c|c|c|c|c|c|c|}
\hline$n$ & $\begin{array}{l}r \\
2\end{array}$ & 4 & 6 & 8 & 10 & 12 \\
\hline 0 & -0.780435 & -0.759482 & -0.728325 & -0.694979 & -0.663779 & -0.635945 \\
\hline 1 & -0.0049452 & -0.0226611 & -0.0476133 & -0.0720535 & -0.0923617 & -0.108094 \\
\hline 2 & -0.0005305 & -0.0027410 & -0.0068911 & -0.0126176 & -0.0192286 & -0.026052 \\
\hline 3 & -0.0001405 & -0.0007379 & -0.0019225 & -0.0037146 & -0.0060376 & -0.0087501 \\
\hline 4 & $-5.579 \cdot 10^{-5}$ & -0.0002942 & -0.0007746 & -0.0015242 & -0.0025408 & -0.0037897 \\
\hline 5 & $-2.796 \cdot 10^{-5}$ & -0.0001469 & -0.0003855 & -0.0007604 & -0.0012814 & -0.001952 \\
\hline 6 & $-1.642 \cdot 10^{-5}$ & $-8.691 \cdot 10^{-5}$ & -0.0002332 & -0.0004801 & -0.0008648 & -0.001443 \\
\hline 7 & $-3.10 \cdot 10^{-8}$ & $-8.01 \cdot 10^{-7}$ & $-4.98 \cdot 10^{-7}$ & $-1.73 \cdot 10^{-5}$ & $-4.44 \cdot 10^{-5}$ & $-9.48 \cdot 10^{-5}$ \\
\hline 8 & $-4.13 \cdot 10^{-9}$ & $-1.14 \cdot 10^{-7}$ & $-7.85 \cdot 10^{-7}$ & $-3.06 \cdot 10^{-6}$ & $-8.76 \cdot 10^{-6}$ & $-2.07 \cdot 10^{-5}$ \\
\hline 9 & $-1.15 \cdot 10^{-9}$ & $-3.20 \cdot 10^{-8}$ & $-2.24 \cdot 10^{-7}$ & $-8.95 \cdot 10^{-7}$ & $-2.64 \cdot 10^{-6}$ & $-6.45 \cdot 10^{-6}$ \\
\hline 10 & $-4.01 \cdot 10^{-10}$ & $-1.12 \cdot 10^{-8}$ & $-7.88 \cdot 10^{-8}$ & $-3.20 \cdot 10^{-7}$ & $-9.66 \cdot 10^{-7}$ & $-2.44 \cdot 10^{-6}$ \\
\hline 11 & $-1.44 \cdot 10^{-10}$ & $-4.02 \cdot 10^{-9}$ & $-2.85 \cdot 10^{-8}$ & $-1.17 \cdot 10^{-7}$ & $-3.64 \cdot 10^{-7}$ & $-9.50 \cdot 10^{-7}$ \\
\hline 12 & $-4.30 \cdot 10^{-11}$ & $-1.22 \cdot 10^{-9}$ & $-8.93 \cdot 10^{-9}$ & $-3.84 \cdot 10^{-8}$ & $-1.26 \cdot 10^{-7}$ & $-3.54 \cdot 10^{-7}$ \\
\hline & -0.786151 & -0.786151 & -0.786151 & -0.786151 & -0.786151 & -0.786151 \\
\hline
\end{tabular}

Table 2:

The TCSA coefficients $f_{n}^{(N, r)}$ for $N=12$ and $2 \leq r \leq 12$.

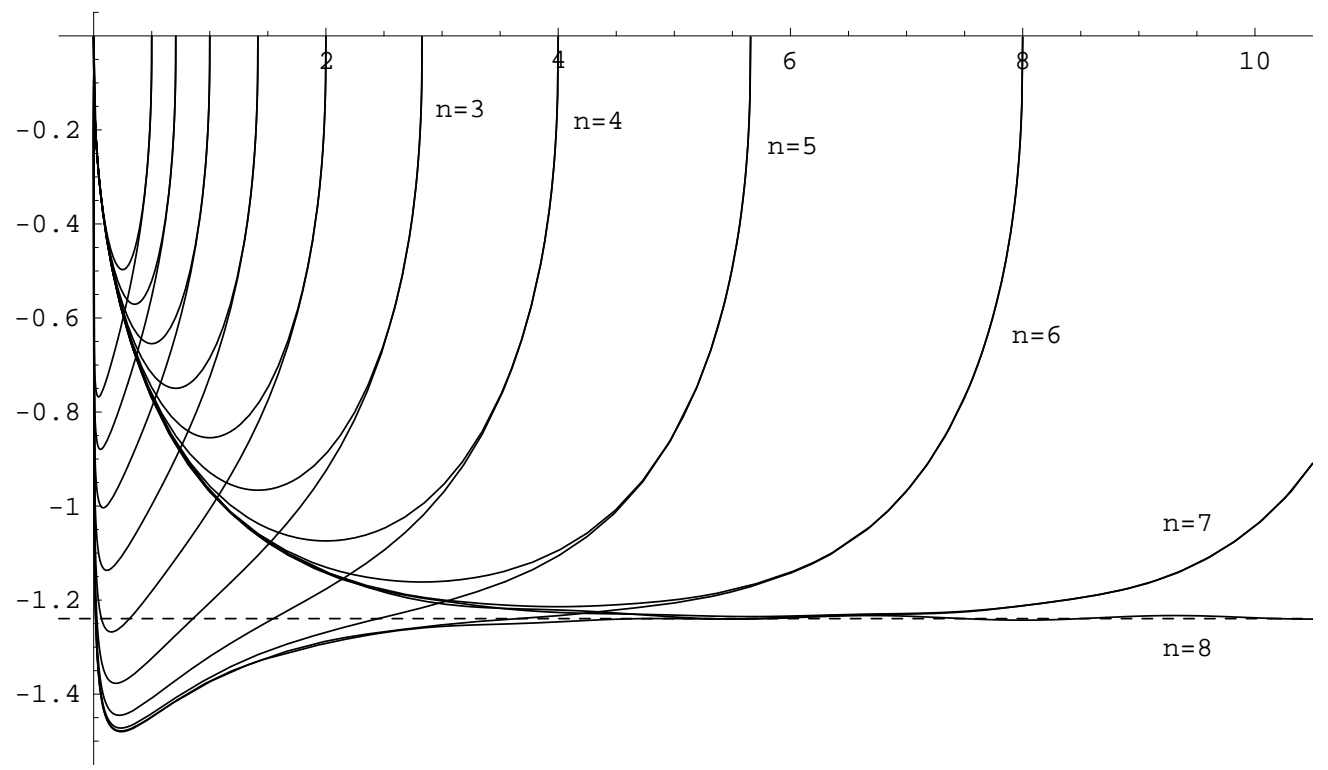

Figure 1

Plots of $\mathcal{G}(\xi)$ and $\mathcal{I}(\xi)$ against $\xi$ from TCSA truncated to level 12. The upper set of lines are the (symmetric) functions $\mathcal{G}(\xi)$, and the lower set of lines are the (asymmetric) functions $\mathcal{I}(\xi)$ for $b=-1 / 2, h=0$. These are plotted for $r=2^{n / 2}$ with $-2 \leq n \leq 8$. As $r$ increases, the functions $\mathcal{G}(\xi)$ and $\mathcal{I}(\xi)$ both approach universal functions which we take to be the expectation values of $M^{2 / 5} \varphi(x)$ on a half-line with boundary conditions $\mathbb{1 1}$ and $\Phi(0)$ respectively. For large $r$, truncation effects start to intervene, as can be seen in the slight 'ripple' discernible for $n=8$. 


\subsubsection{The strip with $(\Phi(h), \mathbb{1})$ boundary conditions}

This case is only slightly more complicated. The unperturbed expectation value is again given in (2.4). The TCSA estimation of the expectation value in the massive model with boundary perturbations then takes the general form

$$
\begin{aligned}
\mathcal{I}^{(N, r)}(\xi) & \equiv M^{2 / 5}\langle\varphi(x)\rangle_{\left.(\Phi(h), \mathbb{1})\right|_{N}} \\
& =\left(\frac{2 r}{\pi}\right)^{2 / 5} \sum_{n=0}^{2 N} \sum_{j=1}^{2}\left(g_{n j}^{(N, r)}(h) f_{j}\left(\frac{\pi \xi}{r}\right) \cos \left(\frac{n \pi \xi}{r}\right)+h_{n j}^{(N, r)}(h) f_{j}^{\prime}\left(\frac{\pi \xi}{r}\right) \sin \left(\frac{n \pi \xi}{r}\right)\right)
\end{aligned}
$$

where $h$ is related to the reflection factor parameter $b$ by $h=h(b)$, and where the functions $g_{n j}^{(N, r)}(h)$ and $h_{n j}^{(N, r)}(h)$ have to be evaluated numerically. Since the ground state $|\widehat{0}\rangle$ of the TCSA Hamiltonian lies in the $h=-1 / 5$ representation, the matrix elements of $\widehat{\varphi}$ are given in terms of the two chiral blocks $f_{1}, f_{2}$; furthermore, since this representation has a null state at level 2, one can eliminate all states with more than one mode $L_{-1}$, which leads to the fact that one does not need to use higher than the first derivative of the chiral blocks in (3.9). Since there are rather many coefficients $g_{n}^{(N, r)}, h_{n}^{(N, r)}$, we shall not give any explicit examples.

In figure 1 we also plot $\mathcal{I}(\xi)$ against $\xi$ for various values of $l$ between 0.5 and 16 , for the fixed value $h=0, b=-1 / 2$. The excellent agreement between $\mathcal{I}(\xi)$ and $\mathcal{G}(\xi)$ that can be seen on the half of the strip $r / 2<\xi<r$ for the larger values of $r$ is a good sign that the TCSA estimates of the functions are converging to their correct values. Table 3 below includes results on the convergence in $N$ of $\mathcal{I}^{(N)}(\xi)$ for values of $\xi$ between 0.01 and 1.0.

\subsubsection{The strip with $\left(\Phi\left(h_{l}\right), \Phi\left(h_{r}\right)\right)$ boundary conditions}

The calculation of the expectation value of $\varphi$ on the strip with two perturbed boundary conditions $\left(\Phi\left(h_{l}\right), \Phi\left(h_{r}\right)\right)$ is in principle the same, except that the functional form is rather more involved. The unperturbed expectation value is again given in (2.4),

$$
\langle\varphi(x)\rangle_{(\Phi(0), \Phi(0))}=\left(\frac{R}{\pi}\right)^{2 / 5}\left({ }^{(\Phi)} B_{\varphi}^{\mathbb{1}} f_{1}\left(\frac{\pi x}{R}\right)+{ }^{(\Phi)} B_{\varphi}^{\phi} C_{\phi \phi}{ }^{\phi} f_{2}\left(\frac{\pi x}{R}\right)\right)
$$

where the functions $f_{i}(\theta)$ are the strip chiral blocks (2.3). However, the massive perturbation introduces terms proportional to the other two chiral blocks and their derivatives, so that the TCSA estimation of the expectation value in the massive model with boundary perturbations takes the general form

$$
\begin{aligned}
\mathcal{H}^{(N, r)}(\xi) & =M^{2 / 5}\langle\varphi(x)\rangle_{\left.\left(\Phi\left(h_{l}\right), \Phi\left(h_{r}\right)\right)\right|_{N}} \\
& =\left(\frac{r}{\pi}\right)^{2 / 5} \sum_{n=0}^{2 N} \sum_{k=1}^{4}\left(j_{k n}^{(N, r)}\left(h_{l}, h_{r}\right) f_{k}\left(\frac{\pi \xi}{r}\right) \cos \left(\frac{n \pi \xi}{r}\right)+k_{k n}^{(N, r)}\left(h_{l}, h_{r}\right) f_{k}^{\prime}\left(\frac{\pi \xi}{r}\right) \sin \left(\frac{n \pi \xi}{r}\right)\right)
\end{aligned}
$$

where $h_{l}=h\left(b_{l}\right), h_{r}=h\left(b_{r}\right)$ and the functions $j_{i n}^{(N, r)}\left(h_{l}, h_{r}\right)$ and $k_{i n}^{(N, r)}\left(h_{l}, h_{r}\right)$ are evaluated numerically. Again, since there are rather many coefficients, we shall not give any explicit examples. For large values of $r$, the two boundaries are essentially non-interacting - this 
was already seen in figure 1. We therefore see no new phenomena over those seen already; the TCSA estimates of $\left\langle M^{2 / 5} \varphi(x)\right\rangle$ near the left boundary from the system with boundary conditions $\left(\Phi\left(h_{l}\right), \Phi\left(h_{r}\right)\right)$ are barely distinguishable from those from the system with boundary conditions $(\Phi(h), \mathbb{1})$. Further confirmation is contained in table 3, which presents data from the two situations.

However, for small $r$, the presence of two perturbed boundaries can destabilise the vacuum even for values of the parameters $h_{l}$ and $h_{r}$ which are less negative than $-\left|h_{\text {crit }}\right|$, the value for which a single boundary destabilises the bulk vacuum on a half line [5]. For $b_{l}+b_{r}=0$, $\left|b_{l}\right|<2$, the ground state and first excited state have an exact crossing at a finite value of $r$, while for $b_{l}+b_{r}>0$ there is a finite range of $r$ for which they become complex. We discuss this in section 6.2, where we give examples of the spectra, and plots of the boundary field expectation values $\left\langle M^{1 / 5} \phi\right\rangle$, for systems with two perturbed boundaries.

\begin{tabular}{|c|c|c|c|c|c|c|c|}
\hline \multirow[b]{2}{*}{$\xi$} & \multicolumn{4}{|c|}{ TCSA truncation level $N$} & \multicolumn{3}{|c|}{ FF truncation level $n$} \\
\hline & 4 & 8 & 12 & 16 & 1 & 2 & 3 \\
\hline 0.01 & $\begin{array}{l}-1.19134 \\
-1.16788\end{array}$ & $\begin{array}{l}-1.16743 \\
-1.16261\end{array}$ & $\begin{array}{l}-1.16362 \\
-1.16234\end{array}$ & $\begin{array}{l}-1.16306 \\
-1.16239\end{array}$ & -1.61719 & -1.21289 & -1.14851 \\
\hline 0.03 & $\begin{array}{l}-1.35236 \\
-1.32317\end{array}$ & $\begin{array}{l}-1.32259 \\
-1.31662\end{array}$ & $\begin{array}{l}-1.31786 \\
-1.31627\end{array}$ & $\begin{array}{l}-1.31716 \\
-1.31633\end{array}$ & -1.60971 & -1.34554 & -1.31311 \\
\hline 0.1 & $\begin{array}{l}-1.48965 \\
-1.45280\end{array}$ & $\begin{array}{l}-1.45199 \\
-1.44456\end{array}$ & $\begin{array}{l}-1.44604 \\
-1.44414\end{array}$ & $\begin{array}{l}-1.44517 \\
-1.44421\end{array}$ & -1.58467 & -1.45438 & -1.44451 \\
\hline 0.3 & $\begin{array}{l}-1.52882 \\
-1.48563\end{array}$ & $\begin{array}{l}-1.48386 \\
-1.47625\end{array}$ & $\begin{array}{l}-1.47722 \\
-1.47586\end{array}$ & $\begin{array}{l}-1.47633 \\
-1.47592\end{array}$ & -1.52209 & -1.47810 & -1.47657 \\
\hline 1.0 & $\begin{array}{l}-1.40584 \\
-1.37786\end{array}$ & $\begin{array}{l}-1.37064 \\
-1.37380\end{array}$ & $\begin{array}{l}-1.37158 \\
-1.37424\end{array}$ & $\begin{array}{l}-1.3738 \\
-1.37447\end{array}$ & -1.37977 & -1.37549 & -1.37546 \\
\hline
\end{tabular}

Table 3:

The TCSA and FF estimates of $\langle\varphi(\xi)\rangle_{\Phi(0)}$, for varying distance $\xi$ from the boundary from the Form-Factor approach truncated at particle number $n$ and from TCSA truncated to level $N$ with $r=12$ on strips with b.c.'s $(\Phi(0), \mathbb{1})$ (upper line) and $(\Phi(0), \Phi(0))$ (lower line). 


\subsection{The comparison of the TCSA and Form-Factor results}

The Form-Factor method gives the expectation value of $\varphi(x)$ on the half-plane. While we can think of the half-plane the infinite-width limit of a finite strip, this is not accessible directly using the TCSA method, which is limited to strips of finite width $r$ and is expected to perform best near to $r=0$. To enable a comparison of the two methods, we shall simply take the TCSA results on a strip of width $r=12$, rather than extrapolating finite width TCSA results to infinite width. (The error for small values of $\xi$ from taking TCSA results at $r=12$ should be much less than the one-particle FF contribution in the middle of the strip, which is $\sim 0.2 \%$ ).

In figure 2 we show results for two cases: the boundary conditions $(\mathbb{1})$ and $(\Phi(0))$. We give the Form-Factor expansion (with $\widetilde{g}=g / 2$ ) truncated to one-, two- and three- particle states, and the TCSA data from truncation to level 16 (with $r=12$ ), and they are clearly in excellent agreement. We also show the Form-Factor expansion assuming $\widetilde{g}=g$, and it is clear that this is wrong. In table 3 we give some numbers illustrating the convergence in TCSA truncation level and in form-factor truncation level for various values of $.01 \leq \xi \leq 1$.

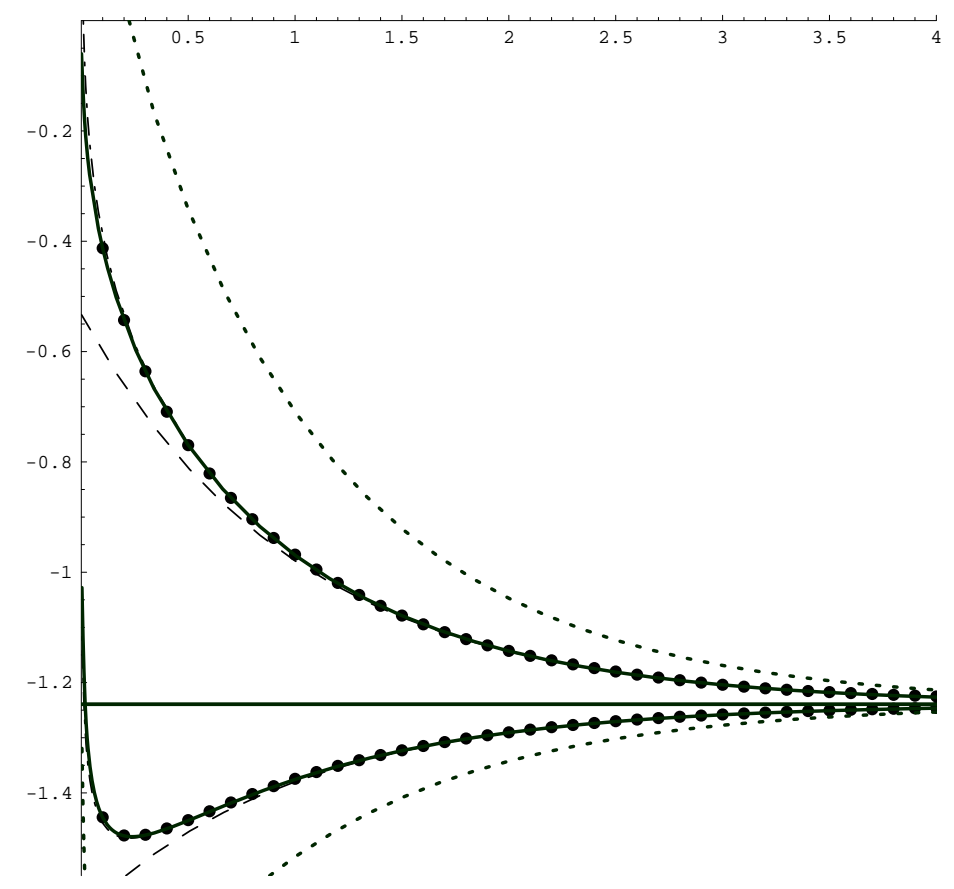

Figure 2

Comparisons of $\left\langle M^{2 / 5} \varphi(x)\right\rangle$ on a half-plane with boundary condition (11) (upper lines) and $(\Phi(0))$ (lower lines). The points are the TCSA data, the dashed lines the FF result up to 1 particle with $\widetilde{g}=g / 2$, the dot-dashed line the FF result up to 2 particles, the solid line the FF result up to 3 particles. The dotted lines are the FF results up to 3 particles with $\widetilde{g}=g$.

The boundary condition $\Phi(0)(b=-1 / 2)$ was chosen because it is for this value of $b$ that the accuracy of the TCSA is highest; comparisons between FF and TCSA results for the further values $b=-1$ and $b=0$ can be found in figure 10, in section 6.1 below. The curve there for $b=0$ is particularly interesting, as this is the case, mentioned at the end of 
section 2 , for which the only difference between the $\Phi(h)$ and the $\mathbb{1}$ boundary states is the sign of the odd-particle-number contributions.

The two- and three- particle form factor expressions in figure 2 are barely distinguishable from the TCSA data, and so in figures 3 and 4 we plot $\log \left(\left\langle M^{2 / 5} \varphi(x)\right\rangle\right)$ against $\log (\xi)$ for the $\mathbb{1}$ and $\Phi(0)$ boundary conditions. We also show the leading behaviour

$$
\begin{aligned}
\left\langle M^{2 / 5} \varphi(x)\right\rangle_{\Phi(0)} & =(2 \xi)^{1 / 5(\Phi)} B_{\varphi}^{\phi}\langle\phi\rangle_{\Phi(0)}+(2 \xi)^{2 / 5(\Phi)} B_{\varphi}^{\mathbb{1}}\langle\mathbb{1}\rangle_{\Phi(0)}+O\left(\xi^{12 / 5}\right) \\
\left\langle M^{2 / 5} \varphi(x)\right\rangle_{\mathbb{1}} & =(2 \xi)^{2 / 5(\mathbb{1})} B_{\varphi}^{\mathbb{1}}\langle\mathbb{1}\rangle_{\mathbb{1}}+O\left(\xi^{12 / 5}\right)
\end{aligned}
$$

of small- $\xi$ expansions obtained from a perturbative treatment of the structure functions. (We intend to report on this approach elsewhere [9].) We see that the three-particle FF approximation already agrees very well with these expansions for $-3 \lesssim \log \xi \lesssim-2$.

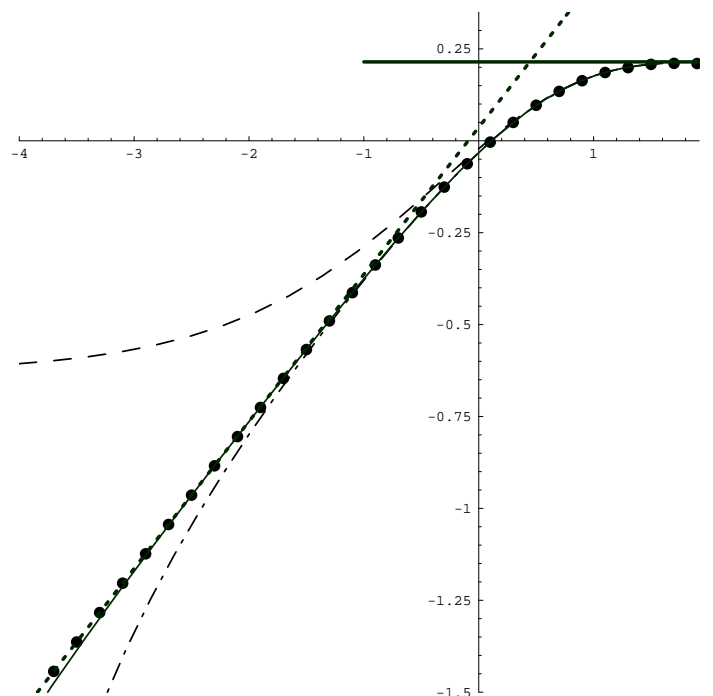

Figure 3:

Plots of $\log \left|\left\langle M^{2 / 5} \varphi(x)\right\rangle_{\mathbb{1}}\right|$ vs. $\log (\xi)$. The points are TCSA data, the dashed, dot-dashed and solid lines are from the FF expansion truncated to one, two and three particles resp., and the dotted line is (3.12).

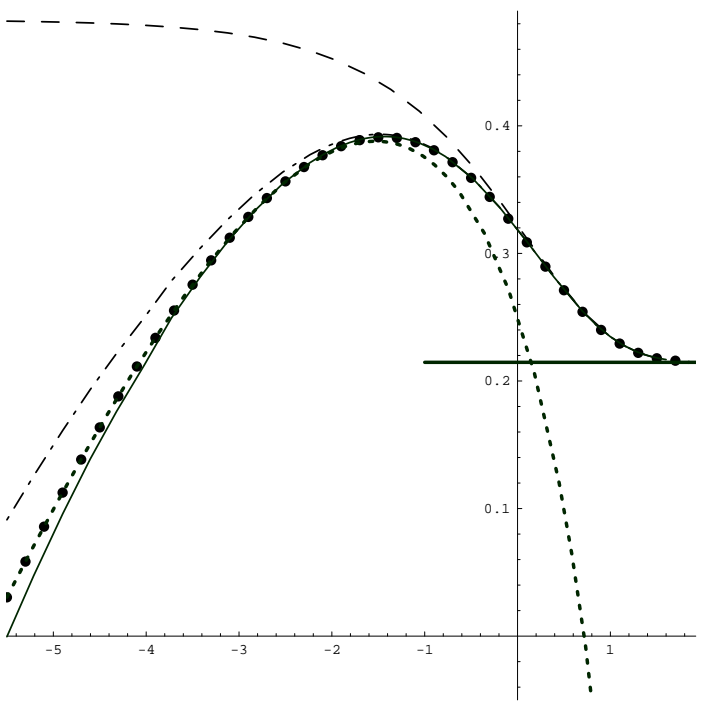

Figure 4:

Plots of $\log \left|\left\langle M^{2 / 5} \varphi(x)\right\rangle_{\Phi(0)}\right|$ vs. $\log (\xi)$. The points are TCSA data, the dashed, dot-dashed and solid lines are from the FF expansion truncated to one, two and three particles resp., and the dotted line is (3.11). 


\section{$4 \quad$ Excited states and energy density}

The TCSA and FF methods are not restricted to the ground state expectation values of the field $\varphi(x, y)$. In this section we give a couple of examples of their wider applicability.

\subsection{The expectation value of $\varphi$ in the first excited state}

It is just as easy in the TCSA to find the first excited eigenstate $|\widehat{1}\rangle$ as to find the ground state and to find the corresponding expectation value of $\varphi$. The result is somewhat less accurate than in the ground state, and this accuracy decreases as higher and higher excited levels are considered. In figure 5 we show (as points) the TCSA result near the $\Phi(0)$ boundary of the strip with boundary conditions $(\Phi(0), \mathbb{1})$ with $r=14$ and truncation level 14 . This state corresponds to the boundary bound state of energy $e_{1}=M \cos ((b+1) \pi / 6)=\sqrt{3} M / 2$, and so we expect that near the $\Phi(0)$ boundary the expectation value is approximately given by the expectation value in the first excited state for the semi-infinite geometry. It turns out that this expectation value can also be obtained using FF techniques, with results that are shown in the various curves on the figure. The calculation relies on an idea of analytic continuation between states; similar methods were used to find TBA equations for excited states in [7].

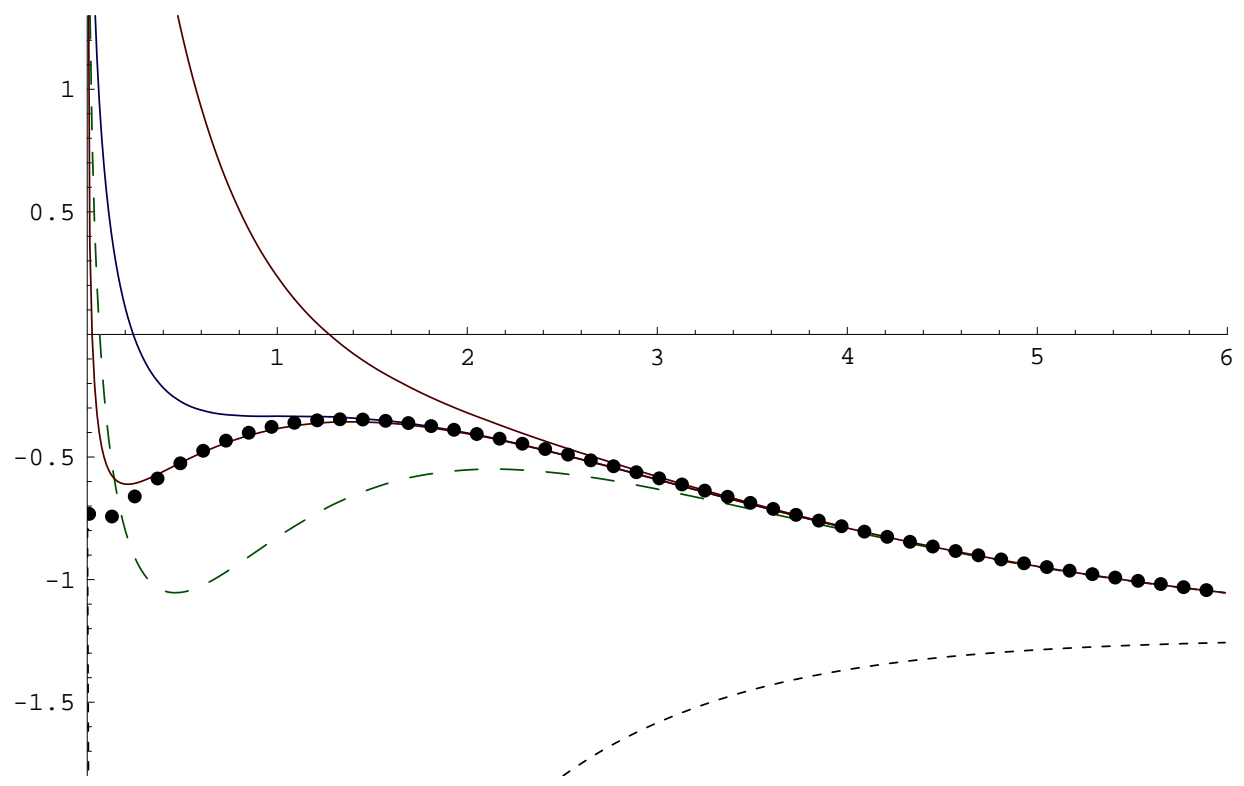

Figure 5:

Comparisons of $\left\langle M^{2 / 5} \varphi(x)\right\rangle_{\Phi(0)}$ in the first excited state. The points are the TCSA data from truncation level 14 and $r=14$. The dotted line is the FF expression (3.6) with $b=9 / 2$; the dashed line is the correction by the terms in (4.2) from the poles at $\theta= \pm(2-b) \pi / 6$, and the solid lines are the results of including all corrections by the addition of the terms up to (4.2), up to (4.3) and up to (4.5) respectively.

The fact that the reflection factors $R_{b}(\theta)$ obey a curious continuation property was already observed in [8]. While the physical parameter $h(b)$ is invariant under under $b \rightarrow 4-b$, the reflection factor $R_{b}$ is not; instead, it is interchanged with the reflection factor of the first 
boundary bound state. This suggests that the expectation value of the field $\varphi$ in the first excited state can be obtained by continuing the FF expression (3.6) from the domain $-3<$ $b<2$ to the region $2<b<5$.

In figure 5 the result of the substitution of $b=9 / 2$ in (3.6) is shown as a dotted line. Clearly, it is a long way from the corresponding TCSA results for $\langle\varphi\rangle^{(1)}$. The explanation is simple: there are poles in $K_{b}(\theta)$ whose positions depend on $b$, in particular at $\theta= \pm i(2-b) \pi / 6$ and $\theta= \pm i(4-b) \pi / 6$. These two pairs of poles cross the integration contour (the real axis) at $b=2$ and $b=4$ respectively, and contributions from both pairs must be added in explicitly to recover the correct analytic continuation in $b$ of $\langle\varphi\rangle$ to $b=9 / 2$, corresponding to $h=0$.

We can regard the contributions from these poles as directly affecting the exponential in (3.4). We denote the positions of the 'active' poles (those which have crossed the integration contour during the continuation) by $\theta_{i}(b)$, and the contribution to the contour integral from the pole in $K_{\alpha}(\theta)$ at $\theta_{i}(b)$ by $k_{i}(b)$ - this will be \pm 1 times the relevant residue, depending on whether the contour was crossed from above or below when the pole became active. Then we can associate the following state in the full-line Hilbert space with the first excited state on the half-line:

$$
\left|B_{\alpha}^{\prime}\right\rangle=\exp \left[\widetilde{g}_{\alpha} A(0)+\frac{i}{2} \sum_{i} k_{i}(b) A\left(-\theta_{i}(b)\right) A\left(\theta_{i}(b)\right)+\int_{-\infty}^{\infty} \frac{\mathrm{d} \theta}{4 \pi} K_{\alpha}(\theta) A(-\theta) A(\theta)\right]|0\rangle .
$$

Expanding this out and inserting the appropriate form factors, the first corrections to the expectation value coming from the residue terms are

$$
\begin{aligned}
& \frac{i}{2} \sum_{i} k_{i}(b)\left\langle 0|\varphi(0)|-\theta_{i}(b), \theta_{i}(b)\right\rangle e^{-2 M x \cosh \theta_{i}(b)}, \\
& \frac{i \widetilde{g}_{\alpha}}{2} \sum_{i} k_{i}(b)\left\langle 0|\varphi(0)| 0,-\theta_{i}(b), \theta_{i}(b)\right\rangle e^{-M x\left(1+2 \cosh \theta_{i}(b)\right)}, \\
&-\frac{1}{4} \sum_{i, j} k_{i}(b) k_{j}(b)\left\langle 0|\varphi(0)|-\theta_{i}(b), \theta_{i}(b),-\theta_{j}(b), \theta_{j}(b)\right\rangle e^{-2 M x\left(\cosh \theta_{i}(b)+\cosh \theta_{j}(b)\right)} \\
& \frac{i}{2} \sum_{i} k_{i}(b) \int_{-\infty}^{\infty} \frac{\mathrm{d} \theta}{4 \pi} K_{\alpha}(\theta)\left\langle 0|\varphi(0)|-\theta_{i}(b), \theta_{i}(b),-\theta, \theta\right\rangle e^{-2 M x\left(\cosh \theta_{i}(b)+\cosh \theta\right)}
\end{aligned}
$$

where the last two terms can be seen as coming from poles in the four-particle contribution to the boundary state which hitherto we have neglected. Also, it turns out that the term (4.4) always gives zero, due to the particular relative positions of the poles at $\theta_{1}$ and $\theta_{2}$.

We have shown the result of correcting (3.4) by the dominant correction (the term in (4.2) coming from the poles at $\theta= \pm(2-b) \pi / 6)$ as a dashed line on figure 5, and the result of adding all terms up to (4.2), up to (4.3) and up to (4.5) as solid lines. It is clear that these are converging rapidly to the TCSA value. 


\subsection{The expectation value of the energy density $\varepsilon$}

As a second example, we use the TCSA to find the expectation values of the energy density $\varepsilon(x)$ on the strip for which

$$
H=\int_{0}^{R} \varepsilon(x) \mathrm{d} x, \quad \varepsilon(x)=-\frac{1}{2 \pi}[T(x)+\bar{T}(x)]+\lambda \varphi(x) .
$$

One has to be rather careful about the specification of the operators in this expression. Here, we mean by $T(x)$ and $\bar{T}(x)$ the 'bare TCSA' quantities - in other words, their expectation values are computed in any given state using the matrix elements of the CFT operators $T$ and $\bar{T}$ between the eigenstates $|\widehat{n}\rangle$ of the perturbed Hamiltonian, themselves expanded in the basis of CFT states. This is the same procedure as was used for computations of $\langle\varphi(x)\rangle$ earlier, but some new issues arise in this case, to which we hope to return in [9].

Leaving these questions to one side, the operator $\varepsilon$ allows us to see directly that 'boundary bound states' are indeed localised at the boundary. In figure 6 we plot the difference in the energy density between the first few excited states and the ground state for the system with boundary conditions $(\Phi(0), \mathbb{1})$ for $r=8$, calculated using TCSA truncated to levels 12,14 and 16. The result of truncating the Fourier-like expansions is very evident here, the TCSA estimates having distinct high-frequency ripples (varying with truncation level) superposed on the overall function.

From the analysis in 5,8, for large $r$ the first excited state is a boundary bound state, and the next several excited states are single-particle scattering states. This is borne out by figure 6, where we see very clearly that the first excited state corresponds to a particle trapped on the left $(\Phi(0))$ boundary and decaying exponentially across the strip, while the higher excited modes are well spread across the strip, attracted to the $\Phi(0)$ boundary and repelled by the $\mathbb{1}$ boundary.

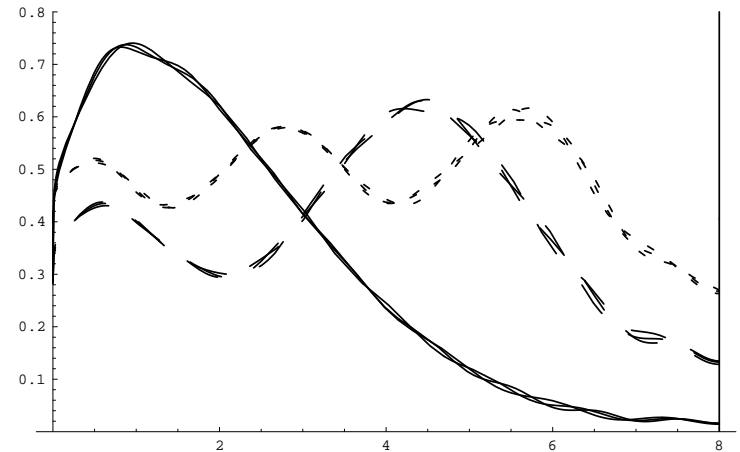

Figure 6: Plots of $M^{-2}\left(\langle\varepsilon(\xi)\rangle_{n}-\langle\varepsilon(\xi)\rangle_{0}\right)$ vs. $\xi$ for the first few excited states on the system with boundary conditions $(\Phi(0), \mathbb{1})$ and $r=8$. The solid line is the 1 st excited state, the dash-dotted line the 2nd, and the dotted line the 3rd. For each line, TCSA results truncated to levels 12, 14 and 16 are superimposed to give an idea of the errors.

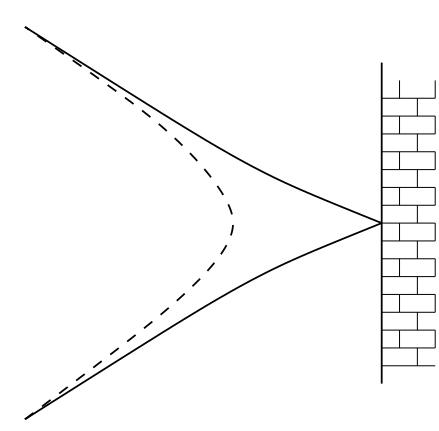

Figure 7: An attractive and a repulsive scattering process with the same classical time delay.

It is impossible to decide whether a boundary $\left(B_{\alpha}\right)$ is repulsive or attractive purely given the 
reflection factors $R_{\alpha}(\theta)$. The $\Phi(h(0))$ and $\mathbb{1}$ boundaries have the same reflection factor but from figure 6 , the $\Phi(h(0))$ boundary is attractive and the $\mathbb{1}$ boundary repulsive. This is the quantum analogue of the inability in a classical theory to determine whether a boundary is attractive or repulsive given only the time-delays - the two processes illustrated in figure 0 have the same time delay but clearly one describes attraction to the boundary and the other repulsion.

\section{The one-point functions of the boundary field}

We now turn to the one-point functions involving the boundary perturbing field $\phi$. In preparation for the main calculations, consider first the partition function $Z_{\alpha \beta}$ of the model on cylinder of length $R$ and circumference $L$, with boundary conditions $\alpha$ and $\beta$ imposed at the two ends. Formally, we can write

$$
Z_{\alpha \beta}=\int[\mathcal{D} \Psi] e^{-\mathcal{A}_{B L Y}}
$$

where $\int[\mathcal{D} \Psi]$ implies that a functional integral over all bulk and boundary degrees of freedom is taken, and $\mathcal{A}_{B L Y}$ denotes the combined bulk and boundary action. For the pair of boundary conditions $(\alpha, \beta)=\left(\Phi\left(h_{l}\right), \Phi\left(h_{r}\right)\right)$, this can be written as

$$
\mathcal{A}_{B L Y}=\mathcal{A}_{B C F T}+\lambda \int_{0}^{R} \mathrm{~d} x \int_{0}^{L} \mathrm{~d} y \varphi(x, y)+h_{l} \int_{0}^{L} \mathrm{~d} y \phi(0, y)+h_{r} \int_{0}^{L} \mathrm{~d} y \phi(R, y)
$$

where $\mathcal{A}_{B C F T}$ is an action for the $\mathcal{M}_{2,5}$ conformal field theory on the cylinder with conformal boundary condition $\Phi$ at the two ends. (For the other two pairs of boundary conditions the expression is similar, but lacks one or both perturbing boundary fields.)

The behaviour of $Z_{\alpha \beta}$ as a function of $R$ and $L$ is complicated, but if both are much larger than all bulk and boundary scales, then, up to exponentially-small corrections,

$$
\log Z_{\alpha \beta} \sim-R L \mathcal{E}_{\text {bulk }}-L f_{\alpha}-L f_{\beta}
$$

where $f_{\alpha}$ and $f_{\beta}$ are the extensive parts of the boundary free energies, and $\mathcal{E}_{\text {bulk }}$ the extensive part of the bulk free energy. For the scaling Lee-Yang model, $\mathcal{E}_{\text {bulk }}=-M^{2} /(4 \sqrt{3})$ [24].

Given $Z_{\alpha \beta}$, the (normalised) one point functions of the field $\phi$ can be simply obtained by differentiation:

$$
\left\langle\phi_{l / r}\right\rangle_{\mathrm{cyl}}=-\frac{1}{L} \frac{\partial}{\partial h_{l / r}} \log Z_{\alpha \beta} .
$$

However, while it was shown in [5] that the partition function was numerically accessible via the TCSA, at the current state of technology $Z_{\alpha \beta}$ (and hence $\langle\phi\rangle_{\text {cyl }}$ ) is not directly computable by means of the TBA. Contact with this 'exact' method can instead be made in certain limits, and these are best discussed using a Hamiltonian formulation.

In fact, there are two alternative Hamiltonian descriptions of the partition function. In the so-called L-channel representation the rôle of time is taken by $L$ :

$$
\begin{aligned}
Z_{\alpha \beta} & =\operatorname{Tr}_{\mathcal{H}_{(\alpha, \beta)}} e^{-L H_{\alpha \beta}(M, R)} \\
& =\sum_{E_{n} \in \operatorname{spec}\left(H_{\alpha \beta}\right)} \exp \left(-L E_{n}^{\operatorname{strip}}(M, R)\right),
\end{aligned}
$$


while in the R-channel representation the rôle of time is taken by $R$ :

$$
\begin{aligned}
Z_{\alpha \beta} & =\left\langle\alpha\left|\exp \left(-R H_{\operatorname{circ}}(M, L)\right)\right| \beta\right\rangle \\
& =\sum_{E_{n} \in \operatorname{spec}\left(H_{\text {circ }}\right)} \frac{\left\langle\alpha \mid \psi_{n}\right\rangle\left\langle\psi_{n} \mid \beta\right\rangle}{\left\langle\psi_{n} \mid \psi_{n}\right\rangle} \exp \left(-R E_{n}^{\operatorname{circ}}(M, L)\right) .
\end{aligned}
$$

In (5.7) we have used the boundary states $|\alpha\rangle$ and $|\beta\rangle$, and the eigenbasis $\left\{\left|\psi_{n}\right\rangle\right\}$ of $H_{\text {circ }}$, the Hamiltonian propagating states living on a circle of circumference $L$. The two decompositions are illustrated in figures 8 and 9 .
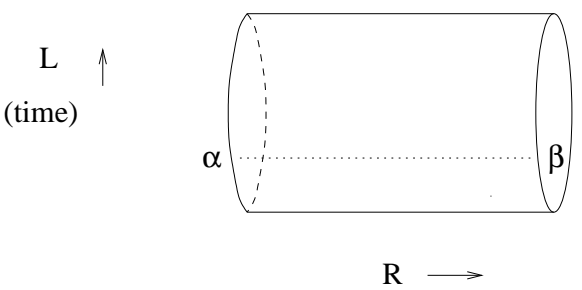

(space)

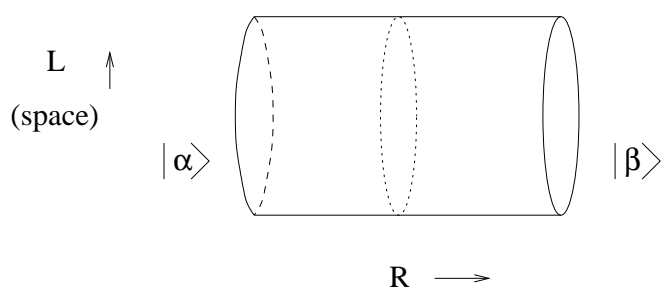

(time)
Figure 8:

The L-channel decomposition: states $\left|\chi_{n}\right\rangle$ live on the dotted line segment across the cylinder.
Figure 9:

The R-channel decomposition: states $\left|\psi_{n}\right\rangle$ live on the dotted circle around the cylinder.

For the rest of this section we will focus on results for the boundary field $\phi_{l}$ on the lefthand end of the cylinder, but with a trivial relabelling it is clear that analogous results for $\phi_{r}$ can be found.

\section{$5.1 \quad$ L-channel decomposition}

First we consider the L-channel representation, depicted in figure 8. This will enable us to find exact formulae for the expectation value of $\phi$ on strips of finite width. Introducing the eigenbasis $\left\{\left|\chi_{n}\right\rangle\right\}$ of $H_{(\alpha, \beta)}$ we differentiate inside the trace (5.5) to find

$$
\left\langle\phi_{l}\right\rangle_{\mathrm{cyl}}=\frac{1}{Z_{\alpha \beta}} \sum_{n} \frac{\left\langle\chi_{n}\left|\phi_{l}\right| \chi_{n}\right\rangle}{\left\langle\chi_{n} \mid \chi_{n}\right\rangle} \exp \left(-L E_{n}^{\mathrm{strip}}(M, R)\right) .
$$

Comparing with (5.6),

$$
\frac{\left\langle\chi_{n}\left|\phi_{l}\right| \chi_{n}\right\rangle}{\left\langle\chi_{n} \mid \chi_{n}\right\rangle}=\frac{\partial}{\partial h_{l}} E_{n}^{\text {strip }}(M, R)
$$

where $\left\langle\chi_{n}\left|\phi_{l}\right| \chi_{n}\right\rangle /\left\langle\chi_{n} \mid \chi_{n}\right\rangle$ is the expectation value of the field $\phi_{l}$ on the left boundary, taken in the $n^{\text {th }}$ excited state.

It was shown explicitly in [5] that, at least for small $n, E_{n}^{\text {strip }}$ can be computed using generalisations of the boundary TBA equations of [16]. For the scaling Lee-Yang model this involves a non-linear integral equation for a single function $\varepsilon(\theta)$ :

$$
\varepsilon(\theta)=2 r \cosh \theta-\log \lambda_{\alpha \beta}(\theta)+\sum_{p} \log \frac{S\left(\theta-\theta_{p}\right)}{S\left(\theta-\bar{\theta}_{p}\right)}-\mathcal{K} * L(\theta),
$$


and an associated set of equations for the (possibly empty) set $\left\{\theta_{p}, \bar{\theta}_{p}\right\}$ of so-called 'active' singularities (cf. [5, 可):

$$
e^{\varepsilon\left(\theta_{p}\right)}=e^{\varepsilon\left(\bar{\theta}_{p}\right)}=-1 \quad(\forall p) .
$$

Here, $r=M R$ as in earlier sections, $L(\theta)=\log \left(1+e^{-\varepsilon(\theta)}\right), f * g(\theta)=\frac{1}{2 \pi} \int_{-\infty}^{\infty} \mathrm{d} \theta^{\prime} f\left(\theta-\theta^{\prime}\right) g\left(\theta^{\prime}\right)$, and, for the $\left(\Phi\left(h\left(b_{l}\right)\right), \Phi\left(h\left(b_{r}\right)\right)\right)$ boundary conditions,

$$
\lambda_{\alpha \beta}(\theta)=K_{b_{l}}(\theta) K_{b_{r}}(-\theta), \quad \mathcal{K}(\theta)=-i \frac{\partial}{\partial \theta} \log S(\theta),
$$

with $K_{b}(\theta)$ defined in (3.2), and $S(\theta)$ the bulk S-matrix (2.7). The number of active singularities depends on the particular energy level under consideration; for some pairs of boundary conditions on the strip it is nonzero even for the ground state [5], in contrast to the situation for the more familiar TBA equations for periodic boundary conditions.

The solution to (5.10) for a given value of $r$ determines the function $c_{n}(r)$ :

$$
c_{n}(r)=\frac{6}{\pi^{2}} \int_{-\infty}^{\infty} \mathrm{d} \theta r \cosh \theta L(\theta)+i \frac{12 r}{\pi} \sum_{p}\left(\sinh \theta_{p}-\sinh \bar{\theta}_{p}\right),
$$

in terms of which $E_{n}^{\text {strip }}(M, R)$ is

$$
E_{n}^{\text {strip }}(M, R)=\mathcal{E}_{\text {bulk }} R+f_{b_{l}}+f_{b_{r}}-\frac{\pi}{24 R} c_{n}(r),
$$

and, rewriting (5.9) in terms of $b_{l}$,

$$
\frac{\left\langle\chi_{n}\left|\phi_{l}\right| \chi_{n}\right\rangle}{\left\langle\chi_{n} \mid \chi_{n}\right\rangle}=\left(\frac{\mathrm{d} h}{\mathrm{~d} b}\right)^{-1} \frac{\partial}{\partial b_{l}} E_{n}^{\text {strip }}=\frac{-5}{\pi\left|h_{\text {crit }}\right| \cos \left(\pi\left(b_{l}+1 / 2\right) / 5\right)} \frac{\partial}{\partial b_{l}} E_{n}^{\text {strip }} .
$$

In the expression (5.14), $f_{b_{l}}$ and $f_{b_{r}}$ are $R$-independent contributions to $E_{n}^{\text {strip }}(M, R)$ from the two boundaries, and $\mathcal{E}_{\text {bulk }}$ is the bulk energy per unit length. If the equations for the particular state under consideration contain active singularities $\left\{\theta_{p}, \bar{\theta}_{p}\right\}$ whose positions do not tend to zero as $r \rightarrow \infty$, then there will be further $R$-independent contributions to $E_{n}^{\text {strip }}(M, R)$ coming from the second term on the RHS of (5.13) - these will be described shortly. However for ground states such contributions are always absent, and so (as anticipated by the notation) $f_{b_{l}}$ and $f_{b_{r}}$ can be identified with the extensive parts of the boundary free energies as defined in (5.3). The exact values of these quantities were extracted from the (ground state) TBA equations in [5], both for the $\Phi(h(b))$ and the $\mathbb{1 1}$ boundaries, and are

$$
f_{b}=\left(\frac{\sqrt{3}-1}{4}+\sin \frac{\pi b}{6}\right) M \quad, \quad f_{\mathbb{1}}=f_{b=0} .
$$

States $\left|\chi_{n}\right\rangle$ lying above the ground state $\left|\chi_{0}\right\rangle$ will generally be separated from $\left|\chi_{0}\right\rangle$ by a finite energy gap, even at large $R$. At the level of the TBA, this gap is seen in the presence of the active singularities, mentioned in the last paragraph, whose positions do not tend to zero as $r \rightarrow \infty$. These give $c_{n}(r)$ a linear growth in $r=M R$, which via (5.14) yields an extra constant term in the large- $R$ asymptotic of $E_{n}^{\text {strip }}(M, R)$. Physically, the gap arises from two sources: the state $\left|\chi_{n}\right\rangle$ may contain a number, $k(n)$ say, of bulk particles bouncing between the two edges of the strip, and in addition there may be a boundary bound state sitting at one 
or both of the boundaries. Suppose that the possible boundary bound states for the left-hand boundary are indexed by $k=0,1, \ldots k_{\max }\left(b_{l}\right)$ with $k=0$ the boundary ground state, and likewise for the right boundary, and that for the state $\left|\chi_{n}\right\rangle$ the left and right boundaries are in states $k_{l}(n), k_{r}(n)$ respectively. Then the general behaviour for $R \rightarrow \infty$ is as follows:

$$
E_{n}^{\text {strip }}(M, R) \sim \mathcal{E}_{\text {bulk }} R+M k(n)+f_{b_{l}}+f_{b_{r}}+e_{k_{l}(n)}\left(b_{l}\right)+e_{k_{r}(n)}\left(b_{r}\right),
$$

where $e_{k}(b)$ is the energy of the $k^{\text {th }}$ boundary bound state of the $\Phi(h(b))$ boundary condition (with $e_{0}(b)=0$, since $k=0$ is just the boundary ground state).

Taking the limit $R \rightarrow \infty$ of the ground state expectation value allows the boundary expectation values on the semi-infinite plane to be recovered. For the state $\chi_{0}, k(0)=$ $k_{l}(0)=k_{r}(0)=0$, and substituting (5.17) into (5.15) using (5.16), we obtain the exact value of the dimensionless expectation value $\left\langle M^{1 / 5} \phi\right\rangle$ in a semi-infinite geometry:

$$
\left\langle M^{1 / 5} \phi\right\rangle=-\frac{5}{6\left|\widehat{h}_{\mathrm{crit}}\right|} \frac{\cos (\pi b / 6)}{\cos (\pi(b+1 / 2) / 5)} .
$$

For now we take $b$ to be restricted to the 'fundamental region' $-3<b<2$, in which case the above formula is indeed correct for the ground state. Its interpretation as $b$ moves outside this region will be described in section 6.1 below.

Returning to finite values of $R$, the asymptotic (5.17) no longer suffices to obtain the expectation value of the boundary field, and one has rather to differentiate the exact formula (5.14), so that (5.18) becomes

$$
\left\langle M^{1 / 5} \phi_{l}\right\rangle(r)=-\frac{5}{6\left|\widehat{h}_{\text {crit }}\right|} \frac{\cos \left(\pi b_{l} / 6\right)-\frac{1}{4 r} \frac{\partial}{\partial b_{l}} c(r)}{\cos \left(\pi\left(b_{l}+1 / 2\right) / 5\right)},
$$

where $\frac{\partial}{\partial b_{l}} c(r) \equiv \frac{\partial}{\partial b_{l}} c_{0}(r)$ can be obtained by differentiating the full TBA equation (5.10) (a similar idea was applied to the case of periodic boundary conditions in 24]). Restricting, for simplicity, to the ground state energy in the region where no active singularities are present, the terms involving the $\theta_{p}$ in $(5.10)$ are absent, and

$$
\frac{\partial}{\partial b_{l}} c(r)=-\frac{6}{\pi^{2}} \int_{-\infty}^{\infty} \mathrm{d} \theta r \cosh \theta \frac{\eta(\theta)}{1+\exp (\varepsilon(\theta))}
$$

where $\varepsilon(\theta)$ solves (5.10), while $\eta(\theta)=\frac{\partial}{\partial b_{l}} \varepsilon(\theta)$ can be obtained from the linear integral equation

$$
\eta(\theta)=-\frac{\partial}{\partial b_{l}} \log \lambda_{\alpha \beta}(\theta)+\mathcal{K} * \frac{\eta}{1+\exp (\varepsilon)}(\theta) .
$$

In this way the final estimates for $\eta(\theta)$ and $\varepsilon(\theta)$ have roughly the same accuracy.

We also estimated $\langle\phi\rangle$ using the TCSA. For this, we used a strip geometry with specific boundary conditions $(\alpha, \beta)$ on the two edges. We then calculated the dimensionless expectation value $\left\langle M^{1 / 5} \phi_{l}\right\rangle_{\alpha, \beta}(r)$, as a function of the strip width $r$ for finite truncation level $N$. While $\left\langle M^{1 / 5} \phi_{l}\right\rangle_{\alpha, \beta}$ can depend strongly on $\beta$ for small $r$, as $r$ increases, this dependence decreases, and as $r \rightarrow \infty$ it approaches the half-plane value $\left\langle M^{1 / 5} \phi\right\rangle_{\alpha}$. In table 0 we compare 
the TCSA estimates for $\left\langle M^{1 / 5} \phi\right\rangle_{\alpha, \beta}$ for $\alpha=\Phi(0), \beta=\mathbb{1}$ and $r$ small with the numerical solution of the ground state TBA equations (5.9,5.10,5.11) and (5.20,5.21). A similar agreement was found for other pairs of boundary conditions.

In table 5 we also give $\left\langle M^{1 / 5} \phi\right\rangle$ for the same boundary conditions for larger values of $r$ to show the convergence to the IR value, and include several plots of $\left\langle M^{1 / 5} \phi\right\rangle$ for various boundary conditions in figure 14 .

\begin{tabular}{r|ccccc} 
& \multicolumn{5}{c}{$\log (r)$} \\
& -8 & -6 & -4 & -2 & 0 \\
\hline TCSA & -0.19684202180 & -0.29365378867 & -0.4380797960 & -0.65350591 & -0.969216 \\
TBA & -0.19684202181 & -0.29365378869 & -0.4380797961 & -0.65350592 & -0.969217 \\
\hline
\end{tabular}

Table 4 :

The TCSA estimates, with truncation level $N=14$, of $\left\langle M^{1 / 5} \phi\right\rangle_{\alpha, \beta}$ for $\alpha=\Phi(0), \beta=\mathbb{1}$ in the small $r$ region compared with the numerical prediction from the TBA

\begin{tabular}{|c|c|c|c|c|c|c|}
\hline & \multicolumn{5}{|c|}{$\log (r)$} \\
\hline & & 0 & 1 & $3 / 2$ & 2 & $5 / 2$ \\
\hline \multirow{10}{*}{$N$} & & -0.969212 & -1.129791 & -1.166399 & -1.175226 & -1.182737 \\
\hline & 0 & -1.321108 & -1.202314 & -1.179004 & -1.174604 & -1.175250 \\
\hline & & -0.969214 & -1.129788 & -1.166291 & -1.174404 & -1.178406 \\
\hline & 8 & -1.321116 & -1.202350 & -1.179061 & -1.174612 & -1.174563 \\
\hline & & -0.969215 & -1.129795 & -1.166281 & -1.174229 & -1.176402 \\
\hline & 10 & -1.321120 & -1.202370 & -1.179099 & -1.174643 & -1.174357 \\
\hline & 1 & -0.969216 & -1.129801 & -1.166281 & -1.174121 & -1.175346 \\
\hline & 12 & -1.321122 & -1.202382 & -1.179124 & -1.174679 & -1.174337 \\
\hline & 14 & -0.969216 & -1.129806 & -1.166288 & -1.174104 & -1.175196 \\
\hline & 14 & -1.321124 & -1.175194 & -1.179142 & -1.174709 & -1.174342 \\
\hline
\end{tabular}

Table 5:

The TCSA estimates of $\left\langle M^{1 / 5} \phi\right\rangle_{\Phi(0), \mathbb{1}}(r)$ (upper data) and $\left\langle M^{1 / 5} \phi\right\rangle_{\Phi(0), \Phi(0)}(r)$ (lower data). These can be compared with the 'exact' IR value of $-1.17459499975 \ldots$ 


\section{$5.2 \quad$ R-channel decomposition}

We now turn to the R-channel representation, depicted in figure 9. This will ultimately lead to expressions for expectation values when the boundary field is placed at one end of a semiinfinite cylinder. We shall use the notation of [6] and denote the boundary states $|\alpha\rangle$ and $|\beta\rangle$ as $\left|\Phi\left(h_{l}\right)\right\rangle$ and $\left|\Phi\left(h_{r}\right)\right\rangle$ respectively.

If $R$ is taken to infinity in (5.7) with all other variables held fixed, then the contribution of the ground state $\left|\psi_{0}\right\rangle \equiv|\Omega\rangle$ will dominate the spectral sum. Thus

$$
Z_{\alpha \beta} \sim A_{\alpha \beta}(M, L) \exp \left(-R E_{0}^{\operatorname{circ}}(M, L)\right)
$$

where $E_{0}^{\text {circ }}(M, L)$ is the ground state energy of $H_{\text {circ }}$ and

$$
A_{\alpha \beta}(M, L)=\frac{\langle\alpha \mid \Omega\rangle\langle\Omega \mid \beta\rangle}{\langle\Omega \mid \Omega\rangle} .
$$

If we now let $L$ grow as well and compare with (5.3), we see that the inner products appearing in (5.23) will, in general, contain a term corresponding to a boundary free energy per unit length:

$$
\log \left(\frac{\langle\Omega \mid \alpha\rangle}{\langle\Omega \mid \Omega\rangle^{1 / 2}}\right)=-L f_{\alpha}+\log \left(g_{\alpha}(M, L)\right) .
$$

On the other hand, this linear term can be extracted from the small- $L$ behaviour of the functions $\log \left(g_{\alpha}(M, L)\right)$, for which 'L-channel' TBA equationsil were proposed in 16. This is explained in [6], where a precise match with the earlier result (5.16) was found. The consistency between these two determinations of $f_{b}$ is in some respects a mystery, since, from other results reported in [6], there are good reasons to doubt the ability of the L-channel TBA equations of [16] to describe the full variation of $\log \left(g_{\alpha}(M, L)\right)$ as a function of $M L$.

Returning to the R-channel decomposition of the full partition function, we have

$$
\left\langle\phi_{l}\right\rangle_{\mathrm{cyl}}=-\frac{1}{L Z_{\alpha \beta}} \sum_{n} \frac{\mathrm{d}}{\mathrm{d} h_{l}}\left\langle\Phi\left(h_{l}\right) \mid \psi_{n}\right\rangle \frac{\left\langle\psi_{n} \mid \Phi\left(h_{r}\right)\right\rangle}{\left\langle\psi_{n} \mid \psi_{n}\right\rangle} \exp \left(-R E_{n}^{\mathrm{circ}}(M, L)\right)
$$

The following identification was made in the massless [1] and massive [6] cases:

$$
\left\langle\Phi(h(b)) \mid \psi_{n}\right\rangle=Y_{n}\left(i \pi \frac{b+3}{6}\right)\left\langle\mathbb{1} \mid \psi_{n}\right\rangle,
$$

where $Y_{n}(\theta)=\exp \left(\varepsilon_{n}(\theta)\right)$, and $\varepsilon_{n}(\theta)$ is the solution of the excited-state TBA equation (with periodic boundary conditions) for the state $\left|\psi_{n}\right\rangle$ (see [2, []). Taking the limit $R \rightarrow \infty$ one deduces that

$$
\left\langle\phi_{l}\right\rangle=\frac{\left\langle\Phi(h)\left|\phi_{l}\right| \Omega\right\rangle}{\langle\Phi(h) \mid \Omega\rangle}=-\frac{1}{L}\left(\frac{\mathrm{d} h}{\mathrm{~d} b}\right)^{-1} \partial_{b} \log Y\left(i \pi \frac{b+3}{6}\right)
$$

which gives the 1-point expectation value of $\phi$ acting on the end of an infinite cylinder of circumference $L$ in terms of the function $Y$. One check on this formula is easily made: from the large- $L$ limit of the TBA equation for $Y$, we have

$$
\log Y\left(i \pi \frac{b+3}{6}\right) \sim-M L \sin \frac{\pi b}{6}=-L\left(f_{b}-f_{\mathbb{1 1}}\right) \quad(L \rightarrow \infty)
$$

\footnotetext{
'the terminology is unfortunately, but unavoidably, a little confusing - these equations are called 'L-channel' because their derivation proceeds via the L-channel representation of the partition function.
} 
using (5.16) in the second equality; differentiating, the expectation value on the upper halfplane quoted in (5.18) is recovered.

There is evidence (see [7]) that the full set of "excited" $Y_{n}$ 's can be obtained from $Y$ via a process of analytic continuation in the bulk perturbing parameter $\lambda$. It then seems reasonable to suppose that (5.27) can also be continued, leading to the following general relation:

$$
\left\langle\phi_{l}\right\rangle^{(n)}=\frac{\left\langle\Phi(h)\left|\phi_{l}\right| \psi_{n}\right\rangle}{\left\langle\Phi(h) \mid \psi_{n}\right\rangle}=-\frac{1}{L}\left(\frac{\mathrm{d} h}{\mathrm{~d} b}\right)^{-1} \partial_{b} \log Y_{n}\left(i \pi \frac{b+3}{6}\right) .
$$

(Alternatively, one can obtain this simply by differentiating (5.26).)

It turns out that there is a further consequence of the R-channel decomposition. Note first that (5.26) can be used to write the partition function as

$$
Z_{\Phi\left(h\left(b_{r}\right)\right), \Phi\left(h\left(b_{l}\right)\right)}=\sum_{n} \frac{\left\langle\mathbb{1} \mid \psi_{n}\right\rangle\left\langle\psi_{n} \mid \mathbb{1}\right\rangle}{\left\langle\psi_{n} \mid \psi_{n}\right\rangle} Y_{n}\left(i \pi \frac{b_{r}+3}{6}\right) Y_{n}\left(i \pi \frac{b_{l}+3}{6}\right) \exp \left(-R E_{n}^{\mathrm{circ}}(M, L)\right) .
$$

Now, the Y's satisfy the functional relation [27

$$
Y_{n}\left(\theta+i \frac{\pi}{3}\right) Y_{n}\left(\theta-i \frac{\pi}{3}\right)=1+Y_{n}(\theta)
$$

and this suggests the following identity

$$
Z_{\Phi(h(b+2)), \Phi(h(b-2))}=Z_{\mathbb{1}, \mathbb{1}}+Z_{\mathbb{1}, \Phi(h(b))} \cdot
$$

This should hold for all $M, R$ and $L$; from the L-channel decomposition (5.6), it is equivalent to the following relation between the spectra of models on strips of equal widths but different boundary conditions:

$$
\left\{E_{n}^{\text {strip }}(M, R)\right\}_{\Phi(h(b+2)), \Phi(h(b-2))}=\left\{E_{n}^{\operatorname{strip}}(M, R)\right\}_{\mathbb{1}, \mathbb{1}} \cup\left\{E_{n}^{\text {strip }}(M, R)\right\}_{\mathbb{1}, \Phi(h(b))} .
$$

Preliminary numerical work confirms this rather surprising identity, but as yet we do not have a good physical understanding of its origin. However, in section 6.2 below it will be used to formulate an exact conjecture concerning the regions of the $\left(b, b^{\prime}\right)$ plane for which the model with $\left(\Phi(h(b)), \Phi\left(h\left(b^{\prime}\right)\right)\right)$ boundary conditions develops a boundary-induced vacuum instability. 


\section{Applications}

In this section we apply some of the results obtained above to elaborate a few further aspects of the boundary scaling Lee-Yang model. We start with the boundary flows of the semiinfinite system, and then turn to the way that the boundary-induced vacuum instability of the model is affected when the system is confined to a finite strip.

We first recall the way the parametrisations of the TCSA, TBA and FF calculations are related. The physical parameter describing the $\Phi(h)$ boundary is, of course, $h$, which is related to $b$ by

$$
h=-\left|h_{\text {crit }}\right| \sin (\pi(b+1 / 2) / 5) .
$$

This physical parameter $h$ is periodic in $b$ with period 10, and we choose the fundamental domain to be $-3 \leq b \leq 2$. Thus for real $b, h$ is restricted to the range $-\left|h_{\text {crit }}\right| \leq h \leq\left|h_{\text {crit }}\right|$. To reach real values of $h>\left|h_{\text {crit }}\right|$ we can continue in $b$ to values $b=-3+\hat{i b}$ with $\widehat{b}$ real; to reach real values of $h<-\left|h_{\text {crit }}\right|$ we can continue in $b$ to values $b=2+i \widehat{b}$ with $\widehat{b}$ real.

As we pointed out in section 4.1, if we formally continue in $b$ outside the fundamental region we have to be careful, as quantities may not continue naively - in particular the continuation of the 'fundamental' reflection factor to $2<b<5$ in fact describes the reflection properties of the boundary with the addition of (the lowest) boundary bound state.

\subsection{Boundary flows on the semi-infinite system}

It is quite straightforward to see the boundary flow, at the level of the one-point functions, using the TCSA. In [5] the spectra for the strip with boundary conditions $(\Phi(h), \mathbb{1})$ were calculated for several values of $h$, and they are consistent with the idea that this spectrum is real for all real $h>-\left|h_{\text {crit }}\right|$. Thus we can calculate the expectation values of $\varphi(x)$ and $\phi$ on the boundary $\Phi(h)$ by looking at the large $r$ limit of calculations on the strip with bcs $(\Phi(h), \mathbb{1})$. The only restriction is that the TCSA errors increase sharply with $|h|$, so that TCSA results are restricted to a small range of $b$ values centred on $b=-1 / 2$.

The FF and 'exact' (TBA) results have more interesting properties. Recall that the FF calculations are formally functions of

$$
g_{\Phi}(b)=\frac{\tan ((b+2) \pi / 12)}{\tan ((b-2) \pi / 12)} g_{\mathbb{1}}, \quad K_{b}(\theta)=R_{b}\left(i \frac{\pi}{2}-\theta\right) .
$$

The continuation to large positive value of $h$ through $b=-3+\widehat{i b}$ works well, as one can easily verify that none of the $b$-dependent poles in $K_{b}(\theta)$ cross the integration contours in the FF integrals (3.6), and so none of the subtleties described in section 4.1 above arise. The formulae

$$
\lim _{\widehat{b} \rightarrow \infty} g_{\Phi}(-3+i \widehat{b})=g_{\mathbb{1}}, \lim _{\widehat{b} \rightarrow \infty} K_{(-3+i \widehat{b})}(\theta)=K_{0}(\theta)=K_{\mathbb{1}}(\theta),
$$

can therefore be substituted directly into the form factor expansion, establishing the result

$$
\lim _{h \rightarrow+\infty}\left\langle M^{2 / 5} \varphi(x)\right\rangle_{\Phi(h)}=\left\langle M^{2 / 5} \varphi(x)\right\rangle_{\mathbb{1}}
$$

as an exact identity. One can similarly discuss the continuation of the expectation value of the boundary field, finding, as expected,

$$
\lim _{\widehat{b} \rightarrow \infty}\left\langle M^{1 / 5} \phi\right\rangle_{\Phi(h(-3+\hat{i b}))}=0=\left\langle M^{1 / 5} \phi\right\rangle_{\mathbb{1}} .
$$


To illustrate the result (6.3), in figure 10 we plot $\left\langle M^{2 / 5} \varphi(x)\right\rangle$ close to the $\Phi(h)$ boundary for various values of $h$. The FF results smoothly interpolate between the $\mathbb{1}$ and $\Phi(h)$ boundaries, and give good agreement with the TCSA results for small value of $|h|$. The $h \rightarrow \infty$ limit is not directly accessible in TCSA, but we have extrapolated our results in $h$ and we can see that (modulo an amplification of the Fourier-type truncation errors) it shows every sign of converging on the expectation value in the $\mathbb{1} \mathrm{bc}$.

As was discussed in [5], for $h>h(-1)$, in the massive system on the half-plane the $\Phi(h)$ boundary has no boundary bound states, and flows to the $\mathbb{1}$ boundary condition as $h \rightarrow+\infty$. For $h(1)<h<h(-1)$ this boundary has one bound state, and for $-\left|h_{\text {crit }}\right|=h(2)<h<h(1)$ there are two boundary bound states; at $h=-\left|h_{\text {crit }}\right|$ the ground state and first excited states become degenerate and for $h$ less than this critical value $-\left|h_{\text {crit }}\right|$, the system does not have a real vacuum. The presence of the bound states can be understood as particles being trapped near the boundary, as we have seen in section 4.2 .

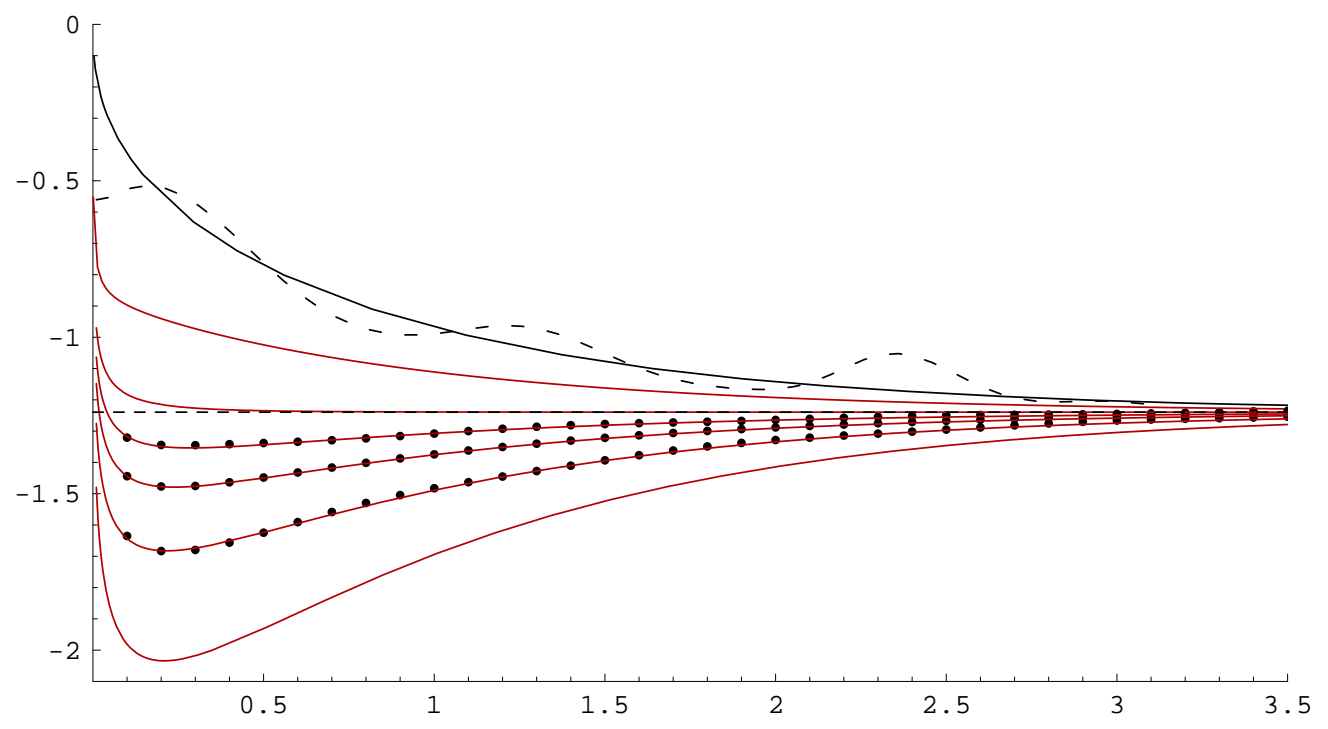

Figure 10

Plots of $\left\langle M^{2 / 5} \varphi(x)\right\rangle_{\Phi(h(b))}$ with boundary condition $\Phi(h(b))$. The solid lines are the Form-Factor results truncated at three particles with $b$ taking values (from second line from top to bottom) $-3+3 i,-2,-1,-1 / 2,0,1 / 2$. The points are the TCSA results $\mathcal{I}(\xi)$ with truncation level 14 and $r=8$. We have also included a plot of $\left\langle M^{2 / 5} \varphi(x)\right\rangle_{\mathbb{1}}$ (top line), the extrapolation of the TCSA data to $h=\infty$ (long dashed line) and the bulk value (short dashed line) for comparison.

The continuation of the FF results beyond $b=2$ was already discussed in section 4.1. The boundary expectation values can also be continued, and here we find a repetition of the reflection factor results - the ground state and first excited state expectation values swap under $b \rightarrow 4-b$, and the second excited state is invariant. To recall, the boundary free energy and the excitation energies of the two lowest lying states (for $1 \leq b \leq 2$ ) are

$$
f_{b}=\left(\frac{\sqrt{3}-1}{4}+\sin (\pi b / 6)\right) M, \quad e_{1}(b)=M \cos (\pi(b+1) / 6), \quad e_{2}(b)=M \cos (\pi(b-1) / 6) .
$$


This gives (cf. eqs. (5.15) and (5.17)) the expectation value $\left\langle M^{1 / 5} \phi\right\rangle^{(n)}$ in these three lowest lying states as

$$
\begin{aligned}
& \left\langle M^{1 / 5} \phi\right\rangle^{(0)}=M^{1 / 5} \frac{\mathrm{d}}{\mathrm{d} h} f_{b}=\left(\frac{5}{6\left|\widehat{h}_{\mathrm{crit}}\right|}\right) \frac{\cos (\pi b / 6)}{\sin (\pi(b-2) / 5)}, \\
& \left\langle M^{1 / 5} \phi\right\rangle^{(1)}=M^{1 / 5} \frac{\mathrm{d}}{\mathrm{d} h}\left(f_{b}+e_{1}\right)=-\left(\frac{5}{6\left|\widehat{h}_{\mathrm{crit}}\right|}\right) \frac{\cos (\pi(4-b) / 6)}{\sin (\pi(b-2) / 5)}, \\
& \left\langle M^{1 / 5} \phi\right\rangle^{(2)}=M^{1 / 5} \frac{\mathrm{d}}{\mathrm{d} h}\left(f_{b}+e_{2}\right)=-\left(\frac{5 \sqrt{3}}{6\left|\widehat{h}_{\mathrm{crit}}\right|}\right) \frac{\sin (\pi(b-2) / 6)}{\sin (\pi(b-2) / 5)} .
\end{aligned}
$$

Note that $\langle\phi\rangle^{(0)}=\langle\phi\rangle^{(1)}$ at the threshold for the first excited state $b=-1$, and $\langle\phi\rangle^{(0)}=$ $\langle\phi\rangle^{(2)}$ at the threshold for the second excited state $b=1$.

Unfortunately the TCSA does not give very good results for the expectation values in the excited states for large $r$, and while extrapolations in $r$ and truncation level indicate that these results are indeed correct, there seems little point in showing any plots.

\subsection{RG flows on the finite size strip}

In [5] the spectrum of the model with $(\mathbb{1}, \Phi)$ boundary conditions was described in some detail, but results for the $(\Phi, \Phi)$ system were not presented. The analysis of these results provides a nice application of the spectral identity found in section 5.2 above, and in this section we describe how this goes. We begin with some results from the TCSA.

In figures $11-13$ the finite size spectrum is plotted for the system on a strip with boundary conditions $\left(\Phi\left(h\left(b_{l}\right)\right), \Phi\left(h\left(b_{r}\right)\right)\right)$ with $b_{l}=b_{r}=b$ taking values $-1 / 2,0,1 / 2$. Observe that the ground and first excited states cross for $b=0$, and for $b=1 / 2$ an interval appears in which the ground state has left the real spectrum. However, so long as $h>-\left|h_{\text {crit }}\right|$ the large- $r$ spectrum stays real.

In figure 14 we plot the $\log$ of the expectation value of the boundary field $\left\langle M^{1 / 5} \phi\right\rangle_{\alpha, \beta}$ against $\log (r)$, for the two pairs of boundary conditions $(\alpha, \beta)=(\Phi(h(b)), \mathbb{1})$ and $(\alpha, \beta)=$ $(\Phi(h(b)), \Phi(h(b)))$ for $b$ taking the values $-1 / 2,0,1 / 2$. For large $r$, the expectation values in the two boundary conditions converge to the same value, as we expect; for large $r$ the influence of the right boundary on the left boundary decreases and the expectation value tends to the half-plane value, $\left\langle\phi_{l}\right\rangle_{\alpha, \beta} \rightarrow_{r \rightarrow \infty}\langle\phi\rangle_{\alpha}$, independent of $\beta$ (provided that the system is not destabilised by the boundary condition $\beta$ ). Conversely, for small $r,\langle\phi\rangle_{\alpha, \beta}$ tend to the conformal limits (2.5) which are governed by the UV fixed point of the boundary flow. On figure 14 the conformal limits are shown as dotted straight lines - the expectation value for $(\alpha, \beta)=(\Phi(h(b)), \mathbb{1})$ all converge to the lower straight line in the UV, and those for $(\alpha, \beta)=(\Phi(h(b)), \Phi(h(b)))$ all converge to the upper line.

The most interesting behaviour is that shown by $\left\langle M^{1 / 5} \phi\right\rangle_{\Phi(h(b)), \Phi(h(b))}$ for intermediate values of $r$. For $b=0$ we saw in figure 12 that the ground state and first excited states cross at one point; at the same point $\langle\phi\rangle$ diverges with a characteristic ' $\lambda$ ' behaviour. For $b=1 / 2$ the ground state drops out of the real spectrum for a finite range of $r ;\langle\phi\rangle$ diverges at the edge points of this range. 


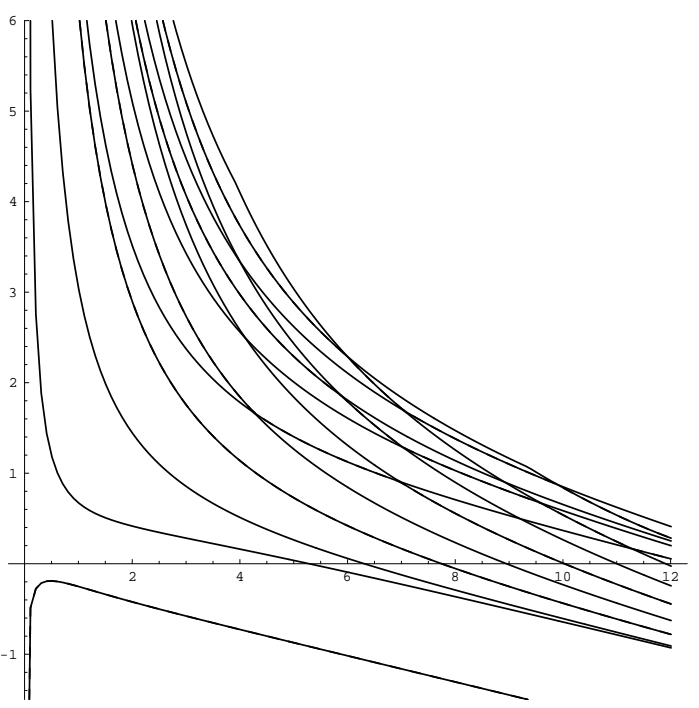

Figure 11: The spectrum of the model on a strip with boundary conditions $b_{l}=b_{r}=-1 / 2$, plotted against $r$.

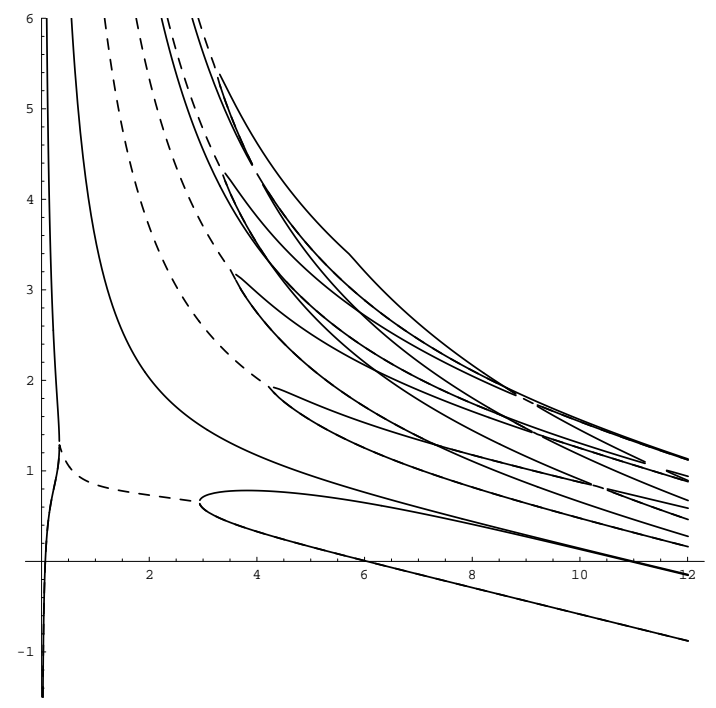

Figure 13: The spectrum of the model on a strip with boundary conditions $b_{l}=b_{r}=1 / 2$, plotted against $r$. The dashed lines indicate the real part of a pair of complex conjugate eigenvalues.

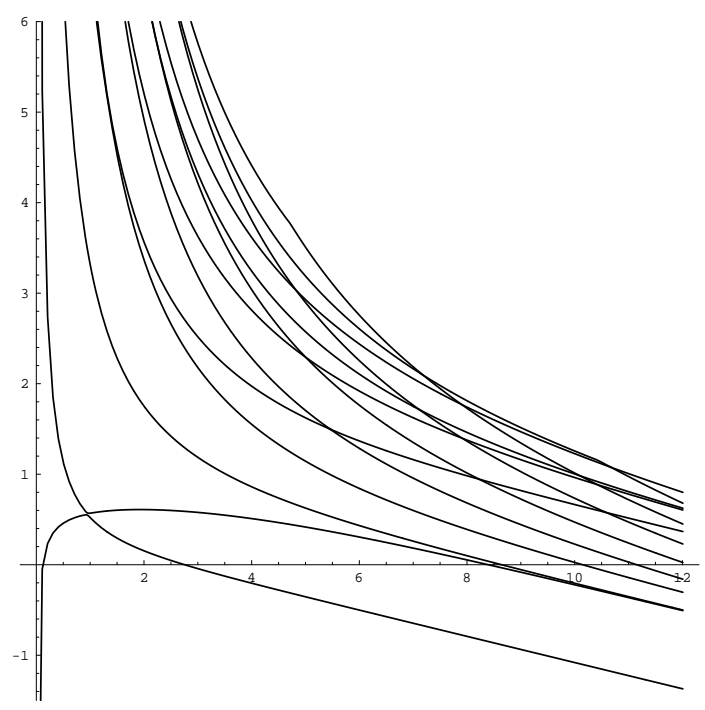

Figure 12: The spectrum of the model on a strip with boundary conditions $b_{l}=b_{r}=0$, plotted against $r$.

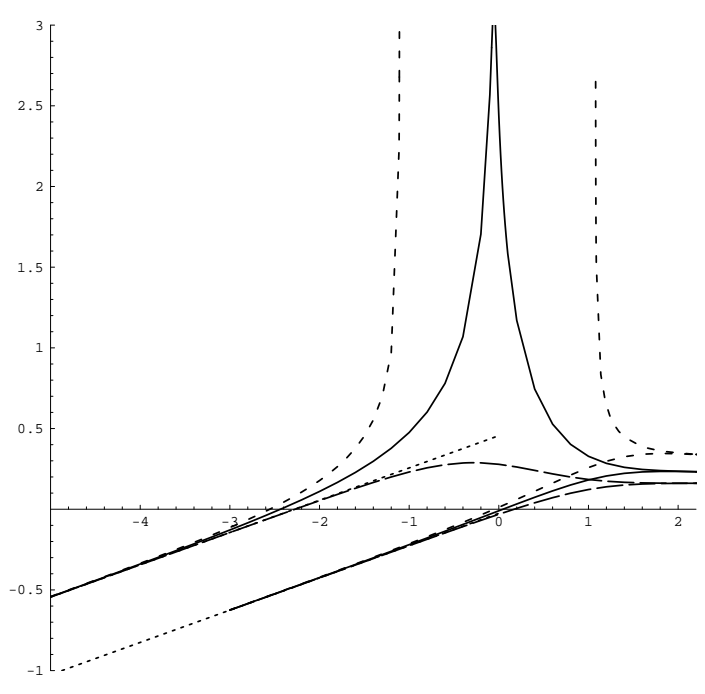

Figure 14: Plots of $\log \left(-\left\langle M^{1 / 5} \phi\right\rangle_{\alpha, \beta}\right)$ against $\log r$ for pairs of boundary conditions $(\Phi(h(b)), \Phi(h(b)))$ and $(\Phi(h(b)), \mathbb{1})$ for $b=-1 / 2$ (long dashed lines), $b=0$ (solid lines) and $b=1 / 2$ (short dashed lines). Also shown (dotted lines) are the conformal expressions (2.5). See text for more details. 
The appearance of level crossing can be understood by making use of the spectral identity (5.33). Shifting $b$, and using the symmetry of the model about $b=2$, the identity can be written as

$$
\left\{E_{n}^{\text {strip }}(M, R)\right\}_{\Phi(h(-b)), \Phi(h(b))}=\left\{E_{n}^{\text {strip }}(M, R)\right\}_{\mathbb{1}, \mathbb{1}} \cup\left\{E_{n}^{\text {strip }}(M, R)\right\}_{\mathbb{1}, \Phi(h(2-b))} .
$$

Consider now the two ground state energies which appear on the RHS of this equation. Depending on their relative values, one or other will correspond to ground state of the model on the LHS. Two limits are easily analysed. As $R \rightarrow 0$, the fact that the $(\mathbb{1}, \Phi)$ conformal Hilbert space contains the field $\psi$ while the $(\mathbb{1}, \mathbb{1})$ does not shows that

$$
\begin{aligned}
\left.\quad E_{0}^{\text {strip }}\right|_{(\mathbb{1}, \mathbb{1})} & \sim+\infty \\
\left.E_{0}^{\text {strip }}\right|_{(\mathbb{1}, \Phi(h(2-b)))} & \sim-\infty
\end{aligned}
$$

In the opposite, large- $R$, limit, the behaviours follow from (5.17):

$$
\begin{aligned}
R \rightarrow \infty:\left.\quad E_{0}^{\text {strip }}\right|_{(\mathbb{1}, \mathbb{1})} & \sim \mathcal{E}_{\text {bulk }} R+(\sqrt{3}-1) M, \\
\left.E_{0}^{\text {strip }}\right|_{(\mathbb{1}, \Phi(h(2-b)))} & \sim \mathcal{E}_{\text {bulk }} R+\left(\sqrt{3}-1+\sin \frac{(2-b) \pi}{6}\right) M .
\end{aligned}
$$

In the latter formula, without loss of generality, we took $b$ to be positive. Comparing (6.5) with (6.6) shows that the relative values of the two ground states on the RHS of (6.4) swap over when going from small to large values of $R$ whenever $b$ is less than 2 . Since these states can be identified with the two lowest-lying levels of the $(\Phi, \Phi)$ model on the LHS of $(\sqrt[6.4]{ })$, we see that a level-crossing in this model is inevitable in all such cases. Thus the point $(0,0)$ in the $\left(b_{l}, b_{r}\right)$ plane, shown in figure 12, belongs to a whole line of points $(b,-b),|b|<2$ which also exhibit a level crossing. The fact that the relevant two states are taken from the spectra of distinct models on the RHS of (6.4) prohibits their mixing and ensures that the crossing will be exact. Once the line $b_{l}+b_{r}=0$ is left, the identity (6.4) can no longer be invoked and the exact level crossing is lost, as can be seen in figures 11 and 13. Observe in the first of these the lowest two levels remain real, while in the second there is an intermediate range of $R$ for which their energies become complex. Physically this can be explained as follows: in the first situation, $b_{l}+b_{r}<0$ and the boundary fields are less strong than in the 'marginal' case of $b_{l}+b_{r}=0$, and hence have less chance to destabilise the model; and in the second the story is reversed, the boundary fields are stronger, and there is therefore a possibility of a vacuum instability for some finite values of $R$. Once this has been understood it is reasonable to conjecture that for all points $\left(b_{l}, b_{r}\right)$ in the fundamental domain $-3 \leq b_{l}, b_{r} \leq 2$ with $b_{l}+b_{r}>0$ the model with $\left(\Phi\left(h\left(b_{l}\right)\right), \Phi\left(h\left(b_{r}\right)\right)\right)$ boundary conditions exhibits a vacuum instability for some range of system sizes, while for the points below this line, the spectrum remains entirely real at all values of $R$.

This picture is confirmed by TCSA plots analogous to figures $11-13$ taken at various other values of $b_{l}$ and $b_{r}$, and leads to the phase diagram for the model shown in figures 15 and 16. The change in coordinates from $\left(b_{l}, b_{r}\right)$ to $\left(h_{l}, h_{r}\right)$ in passing between the two figures transforms the line segment $(b,-b),|b|<2$ into the portion of the ellipse $\left(h_{l}+h_{r}\right)^{2} / \sin ^{2} \frac{\pi}{10}+$ $\left(h_{l}-h_{r}\right)^{2} / \cos ^{2} \frac{\pi}{10}=h_{\text {crit }}^{2}$ on figure 16 which touches the (shaded) region of instability. (Along the rest of the ellipse, the spectral identity (6.4) also holds, but does not imply a level crossing.) Note also that whenever either $h_{l}<h_{\text {crit }}$ or $h_{r}<h_{\text {crit }}$, the vacuum is already 
unstable in infinite volume and so this region can immediately be shaded in, without the need to appeal to more subtle arguments. No markedly new features emerge in the spectra for $b_{l} \neq b_{r}$, so we will not show any further plots. However, we note that the value of $R$ at which the level-crossing occurs for models on the line $b_{l}+b_{r}=0$ diverges as $b_{l}$ (or $b_{r}$ ) tends to 2. This is as one would expect, since in this limit the value of one of the boundary fields is approaching $-\left|h_{\text {crit }}\right|$, and the corresponding boundary bound state is becoming degenerate with the vacuum in infinite volume.

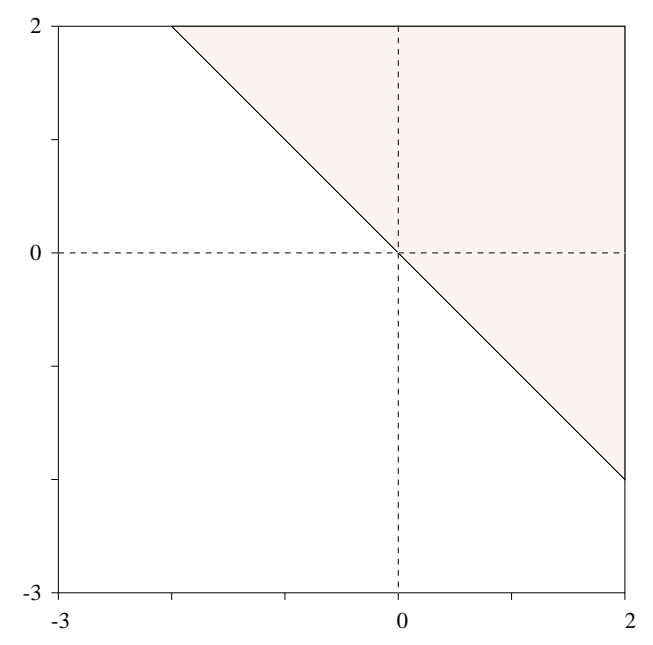

Figure 15:

The fundamental region in the $\left(b_{l}, b_{r}\right)$ plane, showing (shaded) the region within which, for at least one value of the strip width, the model exhibits a boundary-induced instability.

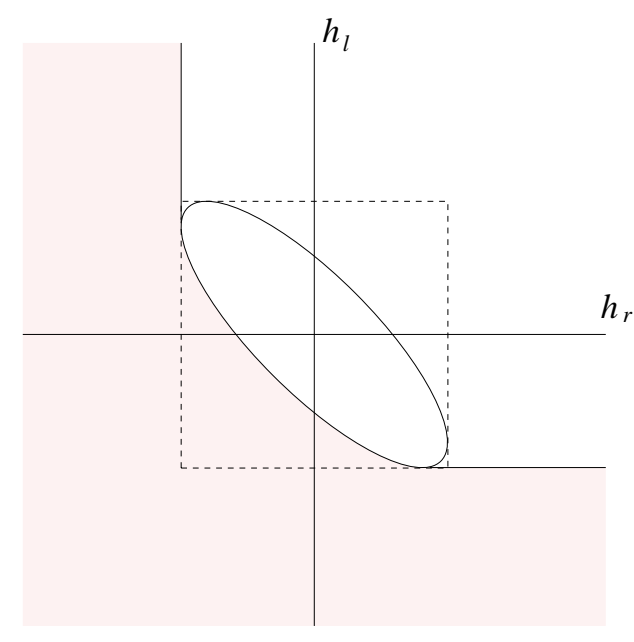

Figure 16:

As figure 15, but plotted on the $\left(h_{l}, h_{r}\right)$ plane. The inner dashed line, a symmetrically-placed square of size $2 h_{\text {crit }}$, indicates the extent of the region covered for real values of $b_{l}$ and $b_{r}$.

\section{Conclusions}

In this paper we have given a rather detailed analysis of the one-point functions of both bulk and boundary fields in a simple but nonetheless non-trivial integrable quantum field theory, the scaling Lee-Yang model. A number of different techniques have been explored, with results which have been shown to be in good accord. Previous work on this topic has tended to use just one method (most usually the form-factor expansion) and thus the consistency that we have found with between the different approaches is an important confirmation that the earlier studies have been well-founded. For this purpose the use of the scaling Lee-Yang model as a testing-ground is very natural, but we feel that it would be very worthwhile to extend this work to further theories, and work on such matters is currently in progress.

There are a number of open questions that arise. The modification to Ghoshal and Zamolodchikov's boundary state that we were forced to make in order to reconcile FF and TCSA results remains a numerical observation, for which we have no terribly compelling physical argument. Furthermore, in section 4.2 we noted that the the boundary states could sometimes be analytically continued, yielding 'excited' boundary states associated with the boundary bound states of the semi-infinite line. A systematic understanding of off-critical boundary states, both in infinite and finite geometries, is still lacking and would presumably 
shed some light on the questions raised by our observations. It also might help to understand at a more fundamental level how the modifications to the boundary TBA equations of [16], found in [5] by an indirect method of analytic continuation, arise.

It would also be worth investigating boundary-to-boundary correlation functions such as

$$
\frac{1}{L^{2}}\left\langle\left(\int \phi_{l}\right)\left(\int \phi_{r}\right)\right\rangle-\left\langle\phi_{l}\right\rangle\left\langle\phi_{r}\right\rangle \text {. }
$$

These are accessible by a simple generalisation of the techniques explained in section 6.1 above, and might be amenable to comparison with, for example, the results of lattice simulations. We also remark that the one-point functions at the end of an infinite cylinder calculated in section 6.2 are essentially the analogues, for the boundary fields of a semi-infinite system, of the finite-temperature expectation values discussed in [15, 18]. In the model we discussed these were relatively easy to obtain as the field under consideration was also the boundary perturbing operator. For more general fields in more complicated models it would presumably be necessary to develop the discussion more along the lines of the work in [18]. This seems to us an important open problem which should certainly be investigated further.

Finally, we remark that the partition function identity discovered in section 6.2 merits further study. In some senses it can be considered as a first off-critical extension of the identities between (sums of) conformal partition functions that can be observed on examining the lists of such objects provided in, for example, [3]. A physical understanding of why such identities should exist is lacking, even in the conformal cases, and perhaps the broader perspective provided by the off-critical results will help towards this end. This alone should motivate the extension our work on off-critical boundary integrable models to further examples.

\section{Acknowledgements -}

The work was supported in part by a TMR grant of the European Commission, contract reference ERBFMRXCT960012, in part by a NATO grant, number CRG950751, and in part by EPSRC grants GR/K30667 and GR/L26216.

PED and GMTW thank the EPSRC for Advanced Fellowships, MP thanks the EPSRC for support and RT thanks the Universiteit van Amsterdam for a post-doctoral fellowship.

We would like to thank Ph. Di Francesco, A. Leclair, G. Mussardo, I. Runkel, G. Takács, Al. Zamolodchikov and J.-B. Zuber for helpful discussions. GMTW would also like to thank R. Guida and N. Magnoli for many discussions of TCSA methods, Brian Davies for very helpful discussions on the numerical analysis of the results, and $\mathrm{SPhT}$ for hospitality during the final stages of this work. 


\section{References}

[1] V.V. Bazhanov, S.L. Lukyanov and A.B. Zamolodchikov, Integrable Structure of Conformal Field Theory, Quantum KdV Theory and Thermodynamic Bethe Ansatz, Commun. Math. Phys. 177 (1996) 381-398, hep-th/9412229.

[2] V.V. Bazhanov, S.L. Lukyanov and A.B. Zamolodchikov, Integrable quantum field theories in finite volume: excited state energies, Nucl. Phys. B489 (1997) 487-531, hep-th/9607099.

[3] R.E. Behrend, P.A. Pearce and J.-B. Zuber, Integrable boundaries, conformal boundary conditions and A-D-E fusion rules, J. Phys. A31 (1998) L763-L770, hep-th/9807142.

[4] J.L. Cardy and G.Mussardo, S matrix of the Yang-Lee edge singularity in two dimensions, Phys. Lett. B225 (1989) 275-278.

[5] P. Dorey, A. Pocklington, R. Tateo and G. Watts, TBA and TCSA with boundaries and excited states, Nucl. Phys. B525 (1998) 641-663, hep-th/9712197.

[6] P. Dorey, I. Runkel, R. Tateo and G. Watts, g-function flow in perturbed boundary conformal field theories, Nucl. Phys. B578 (2000) 85-122, hep-th/9909216.

[7] P. Dorey and R. Tateo, Excited states by analytic continuation of TBA equations, Nucl. Phys. B482 (1996) 639-659, hep-th/9607167;

- Excited states in some simple perturbed conformal field theories, Nucl. Phys. B489 (1998) 575-623, hep-th/9706140.

[8] P. Dorey, R. Tateo and G. Watts, Generalisations of the Coleman-Thun mechanism and boundary reflection factors, Phys. Lett. B448 (1999) 249-256, hep-th/9810098.

[9] P. Dorey, R. Tateo and G. Watts, in preparation.

[10] V.A. Fateev, S. Lukyanov, A.B. Zamolodchikov and Al.B. Zamolodchikov, Expectation values of local fields in Bullough-Dodd model and integrable perturbed conformal field theories, Nucl. Phys. B516 (1998) 652-674, hep-th/9709034.

[11] S. Ghoshal, A.B. Zamolodchikov, Boundary S matrix and boundary state in two-dimensional integrable quantum field theory, Int. J. Mod. Phys. A9 (1994) 3841-3886, erratum Int. J. Mod. Phys. A9 (1994) 4353, hep-th/9306002.

[12] R. Guida and N Magnoli, Vacuum expectation values from a variational approach, Phys. Lett. B411 (1997) 127-133, hep-th/9706017.

[13] R. Konik, A. Leclair and G. Mussardo, On Ising correlation functions with boundary magnetic field, Int. J. Mod. Phys. A11 (1996) 2765, hep-th/9508099.

[14] A. Leclair, F. Lesage and H. Saleur Exact Friedel oscillations in the g=1/2 Luttinger liquid, Phys. Rev. B54 (1996) 13597-13603, cond-mat/9606124.

[15] A. Leclair and G. Mussardo, Finite temperature correlation functions in integrable QFT, Nucl. Phys. B552 (1999) 624-642, hep-th/9902075.

[16] A. Leclair, G. Mussardo, H. Saleur and S. Skorik, Boundary energy and boundary states in integrable quantum field theories, Nucl. Phys. B453 (1995) 581-618, hep-th/9503227.

[17] F. Lesage and H. Saleur, Form-factors computation of Friedel oscillations in Luttinger liquids, J. Phys. A30 (1997) L457-L463, cond-mat/9608112.

[18] S. Lukyanov, Finite temperature expectation values of local fields in the sinh-Gordon model, hep-th/0005027.

[19] G. Mussardo, Sprectral representation of correlation functions in two-dimensional quantum field theories, Talk given at the international colloquium on modern QFT II, Tata Institute, Bombay, January 1994, hep-th/9405128. 
[20] M. Pillin, The form factors in the Sinh-Gordon model, Int. J. Mod. Phys. A13 (1998) 4469-4486, hep-th/9712033.

[21] F.A. Smirnov, The perturbated $C<1$ conformal field theories as reductions of sine-Gordon model, Int. J. Mod. Phys. A4 (1989) 4213-4220;

- Reductions of the sine-Gordon model as a perturbation of minimal models of conformal field theory, Nucl. Phys. B337 (1990) 156-180.

[22] F.A. Smirnov, Form factors in completely integrable models of quantum field theory, Adv. Series in Math. Phys. 14 (World Scientific, Singapore, 1992).

[23] V.P.Yurov and Al.B. Zamolodchikov, Truncated conformal space approach to the scaling Lee-Yang model, Int. J. Mod. Phys. A5 (1990) 3221.

[24] Al.B. Zamolodchikov, Thermodynamic Bethe Ansatz in Relativistic Models. Scaling 3-state Potts and Lee-Yang Models, Nucl. Phys. B342 (1990) 695-720.

[25] Al.B. Zamolodchikov, Two point correlation function in scaling Lee-Yang model, Nucl. Phys. B348 (1991) 619.

[26] Al.B. Zamolodchikov, Mass scale in sine-Gordon model and its reductions, Int. J. Mod. Phys. A10 (1995) 1125-1150.

[27] Al.B. Zamolodchikov, On the thermodynamic Bethe ansatz equations for the reflectionless ADE scattering theories Phys. Lett. B253 (1991) 391-394. 